\title{
Profile and Economic Impacts of Agriculture and Natural Resource Industries in the Northwest Region of Florida
}

\author{
M. Rahmani, A.W. Hodges, and W.D. Mulkey \\ University of Florida/Institute of Food and Agricultural Sciences \\ Food and Resource Economics Department \\ P.O. Box 110240, Gainesville, Fl 32611-0240 \\ Ph. (352)392-1881 X 315 or 312, Fax (352)392-3646 \\ Email: rahmani@ufl.edu or awhodges@ufl.edu
}

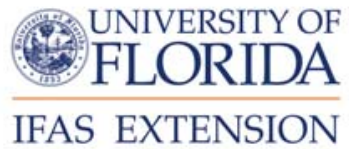

The Institute of Food and Agricultural Sciences (IFAS) is an Equal Opportunity Institution authorized to provide research, educational information and other services only to individuals and institutions that function with non-discrimination with respect to race, creed, color, religion, age, disability, sex, sexual orientation, marital status, national origin, political opinions or affiliations. U.S. Department of Agriculture, Cooperative Extension Service, University of Florida, IFAS, Florida A\&M University Cooperative Extension Program, and Boards of County Commissioners Cooperating. Larry Arrington, Dean 


\section{Executive Summary}

This report provides a profile of agricultural and natural resource industries and an overview of their economic impacts for a 16-county region in northwest Florida based on data from the IMPLAN Pro database for Florida counties for 2002 and data from the 2002 Census of Agriculture. Descriptive data include farm characteristics (number, distribution, farm land use, and investment in land and buildings); value of agricultural products; and measures of economic impacts such as output, valueadded, and employment. Where possible, changes during the1997-2002 time period are highlighted. Additional reports are available for other major regions of Florida.

In 2002, the northwest region of Florida had more than 5,700 farms and over 1 million acres of farmland with an estimated asset value of land and buildings exceeding \$2 billion. Beef and cattle ranching and farming dominated the farm numbers in the region, while woodland not pastured constituted about 310,000 acres, or 30 percent of the reported farmland. Major areas of agricultural activities in the northwest region of Florida included livestock, poultry, milk and dairy products; nursery, greenhouse, and sod; and vegetables, melons and potatoes. The market value of agricultural products sold in the northwest region of Florida exceeded \$275 million in 2002, where the value of all livestock including poultry and their products constituted about $\$ 86$ million, or 31 percent of the regional total. Net farm cash income from farm operations exceeded $\$ 31$ million for all farms in the region for the same period. Jackson County had the highest farm numbers, farm acreage, and estimated asset value of land and buildings, while Gadsden County had the highest value of all agricultural products sold in the northwest region of Florida. Changes in the region during the 19972002 period included a 4 percent decline in the number of farms and an 11 percent decline in cropland acreage. However, the market value of all agricultural products sold decreased only 2 percent between the two census periods. The value of poultry and eggs decreased 43 percent and the value of all livestock and their products decreased by 14 percent during the 1997-2002 time period.

The total economic impacts for all agriculture and natural resource industries in the northwest region of Florida exceeded $\$ 3$ billion in output in 2002. The forestry and related wood and paper products industry was the largest sector, contributing more than $\$ 1.3$ billion in total output impacts and accounting for 44percent of the total in the region. More than 33,000 full- and part-time jobs were generated by agricultural and natural resource industries in the region. Forestry, wood, and paper products, the dominant industry group, produced more than 9,700 jobs and accounted for over 29 percent of total employment impacts for all industry groups in the region. The total value-added impacts of agricultural and natural resource industries exceeded $\$ 1.4$ billion in 2002 . Once again, the forestry, wood, and paper products industry group with $\$ 586$ billion in value-added impacts accounted for 43 percent of the total value-added impacts (the highest for the region). In terms of economic impacts, Escambia County had the highest output impacts, employment impacts, and value-added impacts in the northwest region of Florida. 


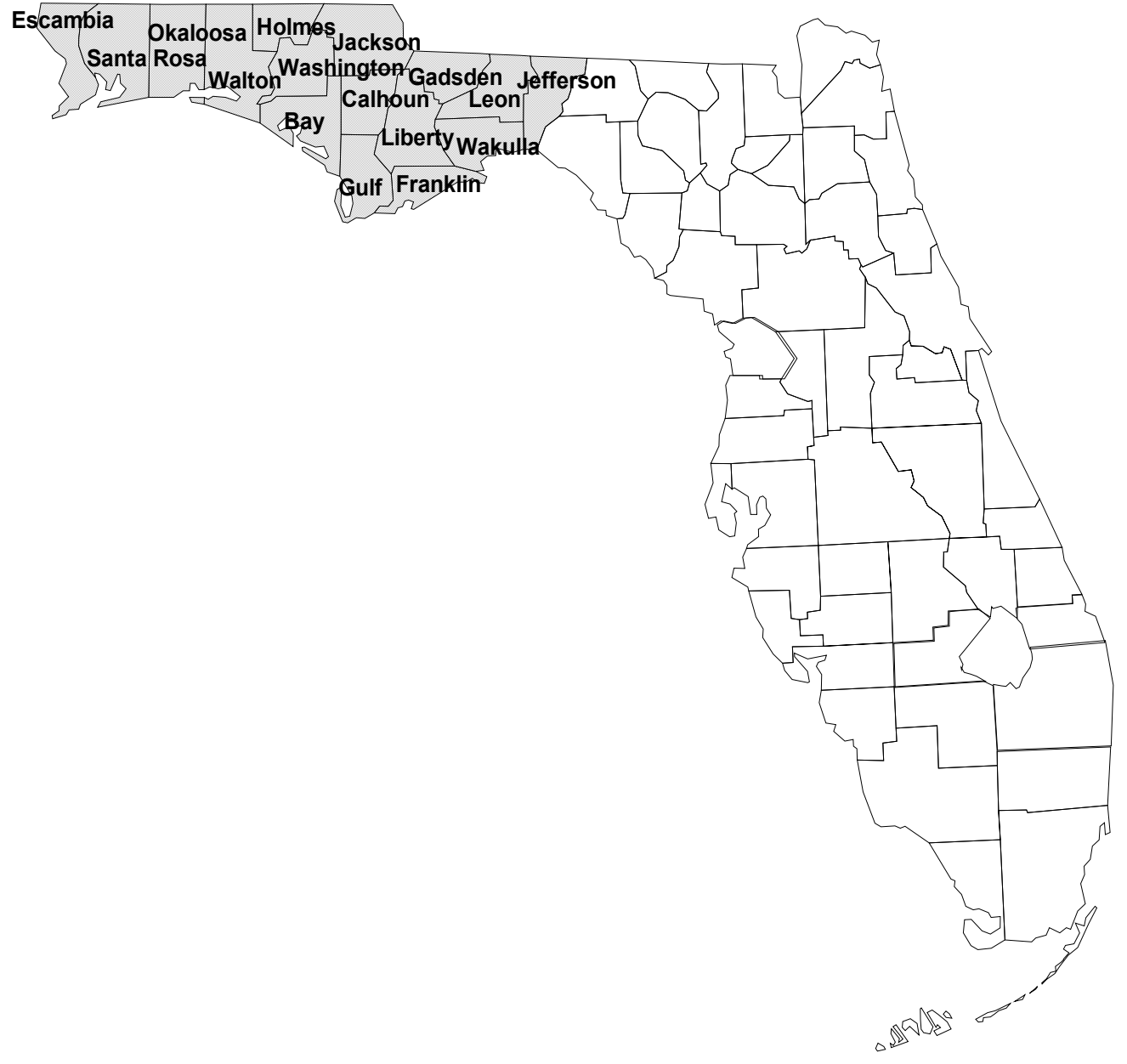

Figure 1. Study area in the northwest region of Florida: Bay, Calhoun, Escambia, Franklin, Gadsden, Gulf, Holmes, Jackson, Jefferson, Leon, Liberty, Okaloosa, Santa Rosa, Wakulla, Walton, and Washington Counties. 


\section{Introduction}

This report provides a profile of agriculture and natural resource industries and an overview of their economic impacts for a 16-county region in northwest Florida (Figure 1). Additional reports provide similar information for the central, northeast, south, and Suwannee River Basin areas of the state. The report is based on data from the IMPLAN Pro database for Florida counties for 2002 and data from the 2002 Census of Agriculture. ${ }^{1}$

The IMPLAN Pro Economic Impact and Social Accounting software package, licensed to the University of Florida by the Minnesota IMPLAN Group, Inc., was used to generate multipliers for output, employment, and value-added for each agricultural and natural resource sector of the regional economy to allow a more comprehensive assessment of impacts. In addition to direct sales of the industries in question, IMPLAN multipliers capture inter-industry purchases (indirect effects) and employee household spending (induced effects). Regional IMPLAN models were constructed for each county using the county-level database provided with the IMPLAN software. No attempt was made to adjust for particular local conditions, and the analysis focused only on economic impacts resulting from operating revenues and employment created by agricultural and natural resource industries.

Data from the 2002 Census of Agriculture ${ }^{2}$ were used to augment the economic impact analysis and to allow for a detailed description of agricultural and natural resource industries for counties in the region. The 2002 Census of Agriculture, conducted by the National Agricultural Statistics Service of the U.S. Department of Agriculture, provides information at the county level on farm numbers; land use; operator characteristics; the market values of land, buildings, and machinery; crop and livestock sales; farm expenses; and income from federal programs.

\section{Farm Characteristics by County}

\section{Farm Numbers (North American Industry Classification System)}

There were over 5,700 farms in the northwest region of Florida in 2002, accounting for 13 percent of all farms in the state (Table 1). Beef and cattle ranching and farming constituted the largest number of farms (1800), or more than 33 percent of all farms in this region. The other crop farming category (tobacco, cotton, hay, and sugarcane) ranked second (1,383 farms), while animal aquaculture and other animal production (961 farms) ranked third. The northwest region had only a few tobacco farms. Jackson County with 920 farms had the highest number of farms in the northwest region (16 percent of all farms in the region). Escambia and Holmes counties each had more than 670 farms.

Farm numbers noted are based on the definition of a farm used by the 2002 Census of Agriculture: "any place from which $\$ 1,000$ or more of agricultural products were produced and sold, or normally would have been sold, during the census year." This definition was first used for the 1974 Census of Agriculture and has remained consistent since so that data are comparable across time periods.

\footnotetext{
${ }^{1}$ IMPLAN Professional, Social Accounting \& Impact Analysis Software, $2{ }^{\text {nd }}$ Edition, June 2000, Minnesota IMPLAN Group, Inc., 1725 Tower Drive West, Suite 140, Stillwater, Minnesota 55082, www.implan.com

2002 Census of Agriculture, Florida, State and County Data, Volume 1, Geographic Area Series, Part 9, AC-02-A-9, Issued June 2004, National Agricultural Statistics Service, U.S. Department of Agriculture.
} 


\section{Farm Size Distribution, Investment in Land, Buildings, and Machinery}

The northwest region had more than 1,014,000 acres of land in farms, accounting for 10 percent of the total farming acreage for Florida (Table 2). Within the region, Jackson County had the largest farm acreage (more than 226,000 acres, or 22 percent of all farmland in the region) and Jefferson County was second in the region (more than 132,000 acres, or 13 percent of the regional total). The largest average farm size was in Calhoun County (325 acres per farm), and Jefferson County ranked second (318 acres per farm). An average farm size of less than 100 acres was reported for Bay, Escambia, and Wakulla Counties.

The estimated asset value of land and buildings for the northwest region exceeded $\$ 2.1$ billion, accounting for 17 percent of total value for all of Florida and for an average value of $\$ 361,000$ per farm in the region. Across the region, Jackson County had the highest value for land and buildings (\$367 million), while Jefferson County had the second highest total (\$244 million). The average value of land and building per farm ranged from close to $\$ 200,000$ in Liberty County upwards to more than $\$ 584,000$ in Jefferson County.

For the region as a whole, the total number of farms decreased by 4 percent, total farm acreage decreased by 1 percent, and total asset values increased by 37 percent during the1997-2002 period. Not all counties, however, followed the regional patterns of change. The number of farms reported in Bay County increased by 17 percent between the two census periods, and smaller percentage increases in farm numbers were reported in Escambia, Liberty, and Okaloosa Counties. Farm numbers remained unchanged in Wakulla County and decreased in all other counties in the region. The number of farms reported in Gulf County decreased by 32 percent between the two census periods, and farm numbers in several counties decreased by more than 10 percent.

Overall farm numbers, however, do not always tell the entire story relative to changes in agriculture at the county level. The increase of 17 percent in the number of farms in Bay County (1997-2002) is a case in point. The increase in farm numbers in Bay County came primarily in the groups of farms in the 1-49 acres category (25 farms) and there was a decrease in farms in the 50-more acres category ( 8 farms). The numbers reflect the increasing popularity of smaller farms.

Across the region, the estimated value of farm investments in land and buildings increased by 37 percent between 1997 and 2002. All counties in the region, except one, had increases in the value of land and buildings, with 9 counties exceeding the average increase for the region. Escambia County had the highest increase (69 percent) in the estimated asset value of land and buildings, while the total value of land and buildings decreased in Franklin County by 8 percent.

The reported farm acreage increased for Bay County by 19 percent (the largest increase), and farm acreage reported in Washington County decreased by 11 percent (the largest decrease). Seven other counties reported increases in farm acreage. As noted earlier, the net effect at the regional level was a modest decline in reported farm acreage over the 1997-2002 period. 


\section{Farmland Use}

Agricultural lands in the northwest region of Florida were used mainly for cropland, nursery, greenhouse, floriculture, pasture, rangeland, and woodland. There were more than 401,000 acres used as cropland in the region in 2002. Harvested cropland constituted only 61 of the total cropland (Figure 2). Nursery, greenhouse, and floriculture included more than 569,000 square feet under glass or other protection plus 3,888 acres in the open. More than 270,000 acres were under all types of pasture, and there were 391,000 acres in farm woodland, with 20 percent of it used as pasture. Land enrolled in conservation or Wetlands Reserve Programs and federal or other crop insurance programs represented a total of 143,000 acres (Tables 3A \& 3B).

For cropland, Jackson County with more than 114,000 acres of cropland ranked first, followed by Santa Rosa and Escambia Counties with 59,000 and 39,000 acres of cropland respectively. Escambia County was by far the largest for nursery, greenhouse, and floriculture, with more than 365,000 square feet under glass or other protection (64 percent of the regional total) in 2002. However, Gadsden County with more than 1,100 acres (29 percent of the regional total) had the highest acreage of nursery, greenhouse, and floriculture in the open.

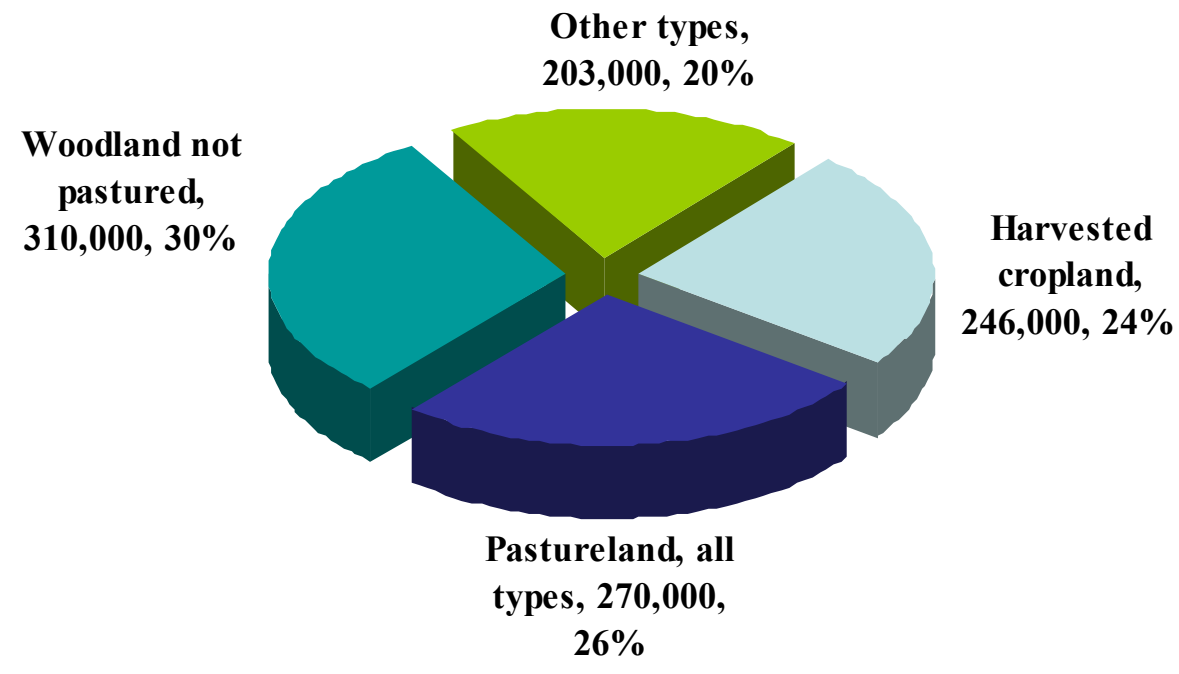

Figure 2. Land use in the northwest region of Florida by acre and percent of total

For the northwest region as a whole, the reported cropland acreage decreased by 11 percent, and pastureland and rangeland acreage increased by 34 percent during the 1997-2002 period. There was, however, significant variation across the region. Reported cropland acreage in 3 counties decreased by a larger percentage than the regional average, with 2 counties reflecting a decrease of more than 30 percent. Reported cropland acreage increased only in 3 counties. Significant increases in cropland acreage were reported for Gulf and Escambia Counties.

Market Value of Agricultural Products Sold, Production Expenses, and Net Farm Cash Income from Operation

Table 4 reports the market value of agricultural products sold in the northwest region of Florida. According to the 2002 Census of Agriculture, "market value of agricultural products sold represents 
the gross market value before taxes and production expenses of all agricultural products sold or removed from the place in 2002 regardless of who received the payment." Market value is equivalent to total sales, including the value of any shares received by partners, landlords, contractors, or others associated with the operation, and the value of commodities placed in Commodity Credit Corporation (CCC) loans. The market value of agricultural products sold does not include payments received for participation in other federal farm programs nor include income from farm-related sources such as custom-work, other agricultural services, or income from nonfarm sources.

The total market value of agricultural products sold in the northwest region of Florida exceeded $\$ 275$ million in 2002 (Table 4), for an average value of more than $\$ 46,000$ per farm. Gadsden County with more than $\$ 266,000$ and Okaloosa County with $\$ 14,000$ per farm market value of all agricultural products sold registered the highest and the lowest value for the region, respectively. Total farm production expenses exceeded \$268 million, for an average of \$42,732 per farm. Net farm cash income from operations in 2002 exceeded \$31 million for all farms in the region. Net farm cash income from operations was negative for 7 counties. Gadsden County registered the highest market value of agricultural products sold in the northwest region, with more than \$91 million (33 percent of the regional total), and it also had the highest net farm cash income from operations (\$29 million, or 95 percent of the regional total). Jackson County with $\$ 36$ million ranked second in the market value of agricultural products sold, but it also had more than $\$ 6$ million loss of income from operation in 2002 (the highest loss in the region). Across the region, market values of products sold increased by only 2 percent between the two census periods, but decreases in total market values were reported in 11 of the 16 counties in the region. Net farm cash incomes reflected declines in 7 counties.

Market value of agricultural products increased over 123 percent in Liberty County and 78 percent in Leon County. In Wakulla and Washington Counties, the market value of agricultural products decreased by 54 and 46 percent, respectively.

\section{Value of Agricultural Products by Commodity Group}

For the northwest region of Florida, the value of livestock, poultry, and their products constituted the highest portion of the market value of agricultural products in 2002, amounting to more than $\$ 85$ million, or 31 percent of the regional total (Table 5). The value of nursery, greenhouse, floriculture, and sod ranked second, with about $\$ 30$ million, or 11 percent of the regional total. The value of vegetables, melons, potatoes, and sweet potatoes ranked third, with $\$ 9$ million (Figure 3). The value of livestock, poultry, and their products in Holmes County exceeded \$27 million (32 percent of the region). Calhoun County had the highest value for nursery, greenhouse, floriculture, and sod products, with more than $\$ 7$ million, or 26 percent of the value of all of these products in the region.

Trends during 1997-2002 showed a 14 percent decrease for the value of livestock, poultry, and their products for the region. The value of all crops, including greenhouse/nursery production, did not change from 1977. Within this broader group, the value of poultry decreased 43 percent, while cattle and calves decreased only 8 percent. When compared to other commodity groups, fruits, tree nuts, and berries represent a smaller total value ( $\$ 4$ million), but their value increased more than threefold during the 1997-2002 period. Various counties in the region, however, did not follow the overall 
trend. Washington County had the highest decrease (40 percent) in value of all crops, including greenhouse/nursery production, while Leon County doubled this value during the 1997-2002 period.

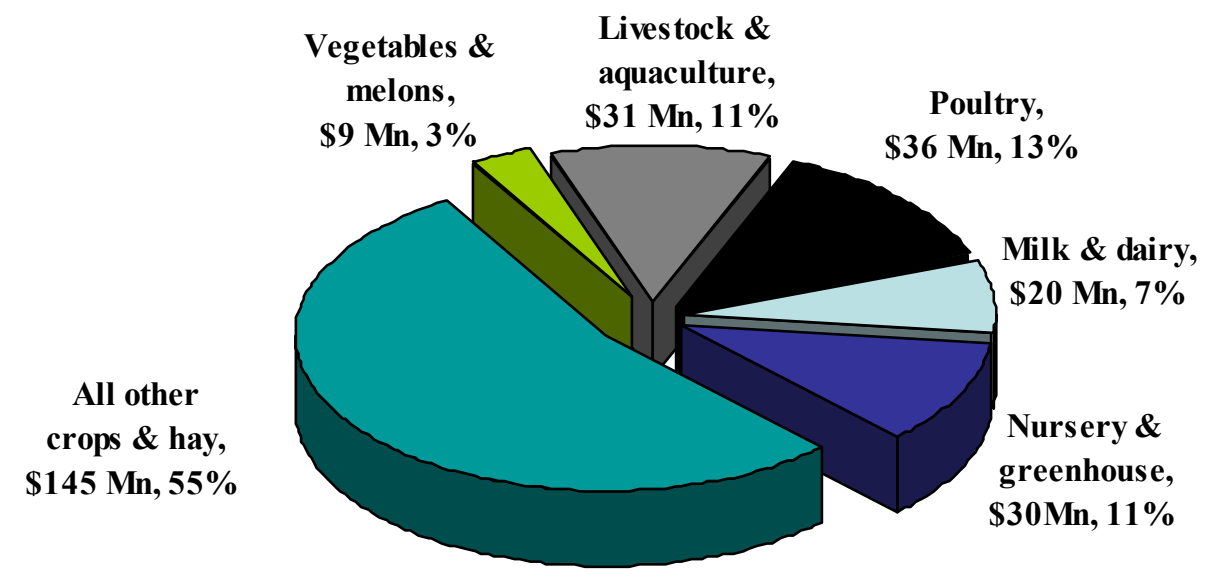

Figure 3. Market value of agricultural products sold in 2002 by commodity group in northwest region of Florida

\section{Principal Operator, Business Organization, and Ethnicity}

In 2002, there were about 8,000 farm operators in the northwest region (29 percent were women). Farming was indicated as the primary occupation by 50 percent of all farm operators; however, in some counties, those listing farming as the primary occupation were less than 50 percent (Table 6). Jackson County with more than 1,100 farm operators accounted for 15 percent (the highest number of farm operators in the northwest region). The number of women operators in Jackson County was also the highest in the region.

Trends during the 1997-2002 period showed that the number of farmers who reported farming as primary occupation in the northwest region increased by 21 percent. During the same time period, the number of farms with farming as the primary occupation increased in Bay and Leon Counties by 86 and 65 percent, respectively.

With respect to the type of business organization, most farms (93 percent) in the northwest region were family and individual types of businesses (Table 7). Partnership farms, corporate farms (family or other than family held), cooperatives, estates or trusts, and institutional farms together comprised only 7 percent of the total number of farms. Family and individual farms exceeded 602,000 acres and accounted for 74 percent of all farm acreage in the northwest region (Figure 4). Corporate farm acreage was about 8 percent of the total for the region; however, most of the corporate farms were family held corporations.

The white ethnic group operated most of the farms in the northwest region. Other ethnic groups were identified as farm operators included Black or African American, Spanish, Hispanic or Latino Origin, American Indian, Alaska Native, Native Hawaiian, other Pacific Islander, and Asian. Whites accounted for 91 percent of all farm operators and operated 97 percent of all farm acreage in the region (Table 8). Some counties reported only the white ethnic group as farm operators. Jackson County had the highest white and minority ethnic farms, farm operators, and acreage. 


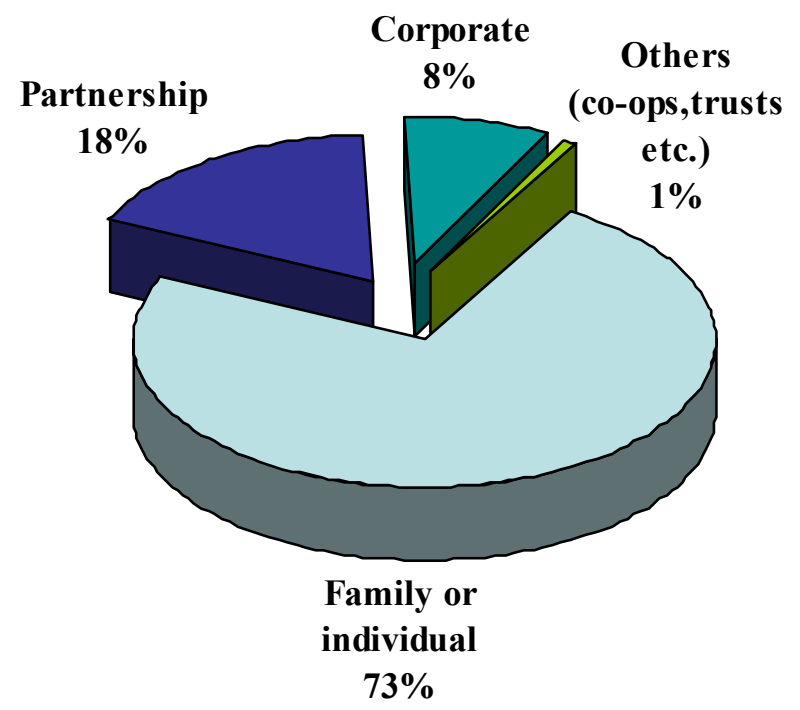

Figure 4. Farm acreage percentages by type of business organization in northwest region of Florida

\section{Economic Impact Results}

As noted earlier, the IMPLAN software package and associated databases allow the estimation of multipliers to capture the total economic impact of agricultural and natural resource industries within a regional or local economy. This section reports the results of impact estimations for counties in the northwest region for each of the agricultural sectors within IMPLAN. Again estimates are based only on the IMPLAN data set with no attempt to adjust for particular local situations and the focus is only on impacts of sales and expenditures associated with farm operations and related manufacturing. ${ }^{3}$

For each reported measure (output, value-added, and employment) for each county, the impacts reported include the direct output of the agricultural sector in question, the impacts associated with indirect inter-industry purchases of inputs by agriculture and its supplying industries, and the induced impacts of spending by employees and owners of agricultural and supplying industries. Reported impacts include activities within production agriculture, natural resource industry (forestry, mining, and fishing), food and forest products manufacturing, agricultural input supply, and agricultural services. Impacts are also reported in terms of labor income and indirect business taxes.

\section{Output Impacts}

Table 9 presents estimates of output impacts for agriculture and natural resource industries by sector and by county for the northwest region of Florida. The total output impacts for all counties in the northwest region exceeded $\$ 3$ billion. Forestry and related wood and paper products was the largest

\footnotetext{
${ }^{3}$ Readers desiring more information on IMPLAN should consult Mulkey \& Hodges, "Using Implan to Assess Local Economic Impacts," Food and Resource Economics Department, UF/IFAS, 2001, http://edis.ifas.ufl.edu/FE168
} 
industry group, contributing more than $\$ 1.3$ billion in total output impacts and accounting for 44 percent of the total in the region. Livestock, dairy farming, and animal product sector with $\$ 407$ million was the next largest industry group in the northwest region. Environmental horticulture (greenhouse/nursery production and landscape services) and mining with a total output impact of $\$ 370$ million and $\$ 230$ million, respectively, were also major contributors to the agriculture and natural resource industries in the northwest region. Fishing and seafood products, grain and oilseed farming and processing, "other" crop farming, and sugarcane farming and refined sugar and confection sectors contributed less to the total output impacts in the region.

In terms of total output impact, Escambia County with more than $\$ 719$ million in output impacts ranked first, and Bay County with $\$ 388$ million ranked second in output impacts in the northwest region. Forestry, wood, and paper products manufacturing with $\$ 562$ million in Escambia County accounted for 42 percent of total output impacts for this industry group in the region and 78 percent of the total output impacts for the county. Gadsden County had the highest output impacts in the fruit and vegetable farming and processing group with $\$ 85$ million, and the highest output impacts for the environmental horticultural industry with $\$ 118$ million. Walton County with more than $\$ 170$ million in output impact of livestock, dairy farming, and animal products registered the highest output impact for this industry group in the northwest region. Santa Rosa County produced more than $\$ 116$ million in output impacts in the mining group (the highest for this sector in the northwest region).

\section{Employment Impacts}

Employment impacts of agriculture and natural resource industries by county and industry group are reported in Table 10. Over 33,400 full- and part-time jobs are generated by agricultural and natural resource industries in the northwest region. Forestry, wood, and paper products manufacturing (the dominant group) produced over 9,700 jobs, or over 29 percent of total employment impacts for the northwest region. The environmental horticulture industry group (greenhouse/nursery production and landscape services) produced over 7,700 jobs. The livestock, dairy farming, and animal products group and the mining group produced more than 4,000, and 3,500 jobs, respectively (Figure 5).

Escambia County dominated most sectors of the agriculture and natural resource industries. With over 6,700 jobs, it accounted for 20 percent of all employment impacts in the northwest region. Gadsden County with over 4,200 jobs ranked second, and Bay County with more than 3,800 jobs ranked third in the number of jobs produced in the region. Santa Rosa County produced more than 2,800 jobs as the result of its strong mining activities (the highest number in the northwest region), and 80 percent of the mining total in the region. Walton County produced 1,362 jobs in the livestock, dairy farming, and animal products industry group (the highest for this group in the region). 


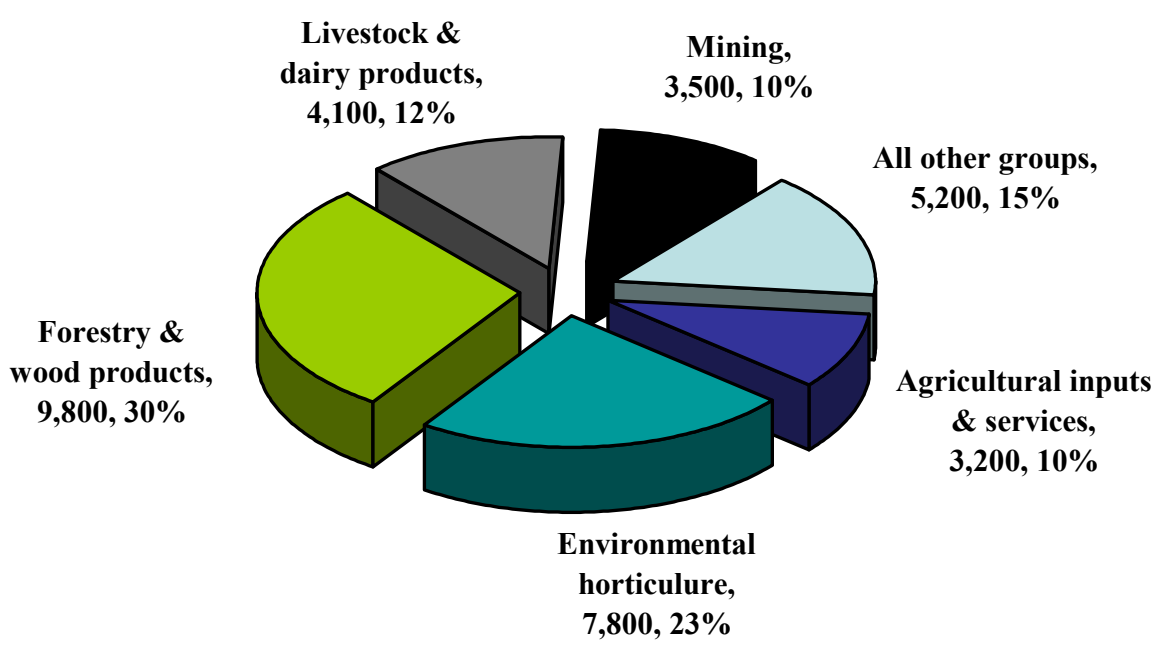

Figure 5. Employment impacts by industry group and the number of jobs in northwest region of Florida

\section{Value-added Impacts}

Value-added is the market value of a firm's output less the value of the inputs which it has purchased from others. It can be viewed as a net economic contribution by the industry sector after direct costs have been subtracted from gross sales. Value-added is the difference between the value of goods as they leave a particular stage of production and the costs of the goods as they entered that stage. Value-added includes payments made by industries to workers, interest, profits, and indirect business taxes. Total value-added impacts of agriculture and natural resource industries in the northwest region exceeded $\$ 1.3$ billion (Table 11). Forestry, wood, and paper product manufacturing with $\$ 586$ million in value-added impacts accounted for 43 percent of the total value-added impacts for the region. Environmental horticulture (greenhouse/nursery production and landscaping services) generated value-added impacts of more than $\$ 253$ million. The mining industry group generated more than $\$ 139$ million in value-added impacts in the northwest region (Figure 6).

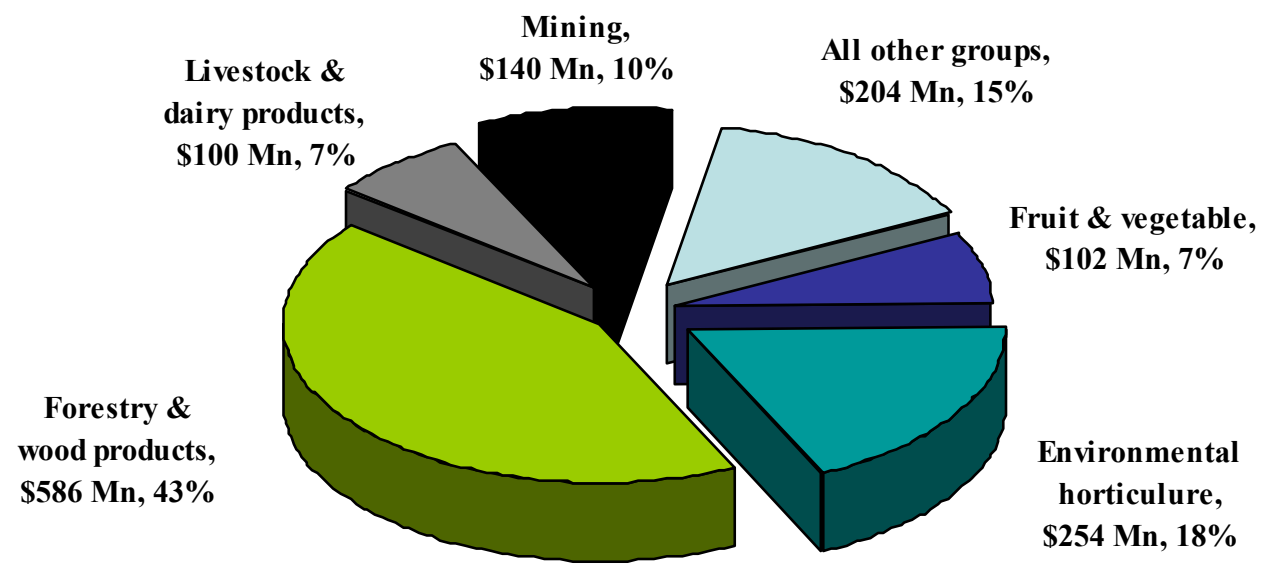

Figure 6. Value-added impacts by industry group in northwest region of Florida 
Escambia County with $\$ 340$ million, accounted for more than 24 percent of the value-added impacts for all counties in the northwest region. Escambia County's dominant position was in the forestry, wood, and paper products group, which accounted for more than \$269 million, or 79 percent of its total value-added impacts. Gadsden County with $\$ 92$ million in value-added impacts in environmental horticulture and Santa Rosa County with \$99 million in value-added impacts in mining topped other counties in those sectors in the northwest region.

\section{Labor Income Impacts}

Labor income impacts totaled about $\$ 874$ million (Table 12). Over 42 percent of the total labor income impact was due to the activities in the area of forestry, wood, and paper products (\$371 million). Other major contributors included environmental horticulture with $\$ 172$ million, agricultural inputs and services with $\$ 86$ million, and mining with $\$ 73$ million.

For labor income impacts, Escambia County was the largest, with more than $\$ 227$ million, or 25 percent of the total labor income impacts for the region, followed by Gadsden County with $\$ 133$ million, and Bay County with $\$ 124$ million in labor income impacts.

\section{Indirect Business Tax Impacts}

Indirect business taxes consist of excise taxes, property taxes, fees, licenses, and sales taxes paid by businesses. These taxes occur during the normal operation of businesses but do not include taxes on profit or income. Total indirect business tax impacts were over \$76 million for the region (Table13). Like the other economic impact measures, forestry, wood, and paper products produced the highest indirect business tax impacts, with $\$ 31$ million, or 40 percent of the total for the region. Once again, Escambia County dominated the northwest regions with about $\$ 19$ million in total indirect business tax impacts, accounting for almost 25 percent of this value for the whole northwest region of Florida.

\section{Acknowledgement}

Constructive comments given by reviewer, Dr. John J. Haydu, are greatly appreciated. 
Table 1. Farms by North American Industry Classification System, northwest region of Florida, 2002

\begin{tabular}{|c|c|c|c|c|c|c|c|c|c|c|c|c|c|c|c|}
\hline County & $\begin{array}{l}\text { Total } \\
\text { farms }\end{array}$ & $\begin{array}{l}\text { Oilseed } \\
\& \text { grain } \\
\text { farming } \\
(1111)\end{array}$ & $\begin{array}{l}\text { Vegetable } \\
\text { '\& melon } \\
\text { farming } \\
(1112)\end{array}$ & $\begin{array}{c}\text { Fruit \& } \\
\text { tree nut } \\
\text { farming } \\
(1113)\end{array}$ & $\begin{array}{l}\text { Greenhouse, } \\
\text { nursery, \& } \\
\text { floriculture } \\
\text { (1114) }\end{array}$ & $\begin{array}{c}\text { Other crop } \\
\text { farming } \\
(1119)\end{array}$ & $\begin{array}{c}\text { Tobacco } \\
\text { farming } \\
(11191)\end{array}$ & $\begin{array}{l}\text { Cotton } \\
\text { farming } \\
(11192)\end{array}$ & $\begin{array}{c}\text { Sugarcane, } \\
\text { hay, \& other } \\
\text { crop } \\
(1119.3 \\
1119.4,1119.9)\end{array}$ & $\begin{array}{c}\text { Beef cattle } \\
\text { ranching } \\
\& \text { farming } \\
(112111)\end{array}$ & $\begin{array}{c}\text { Dairy cattle \& } \\
\text { milk } \\
\text { production } \\
(11212)\end{array}$ & $\begin{array}{c}\text { Hog } \\
\& \text { pig } \\
\text { farming } \\
(1122)\end{array}$ & $\begin{array}{l}\text { Poultry } \\
\& \text { egg } \\
\text { production } \\
(1123)\end{array}$ & $\begin{array}{c}\text { Sheep } \\
\& \text { goat } \\
\text { farming } \\
(1124)\end{array}$ & $\begin{array}{c}\text { Animal } \\
\text { aquaculture } \\
\& \text { other } \\
\text { animal } \\
\text { production } \\
(1125,1129) \\
\end{array}$ \\
\hline Bay & 116 & 2 & 6 & 6 & 11 & 5 & - & - & 5 & 31 & - & 4 & 5 & - & 46 \\
\hline Calhoun & 151 & 5 & 5 & 7 & 9 & 40 & - & 7 & 33 & 45 & 2 & 8 & 1 & 2 & 27 \\
\hline Escambia & 674 & 37 & 20 & 63 & 46 & 142 & - & 24 & 118 & 181 & 9 & 3 & 3 & 11 & 159 \\
\hline Franklin & 20 & - & - & - & - & - & - & - & - & 2 & - & - & 1 & - & 17 \\
\hline Gadsden & 343 & 20 & 14 & 44 & 28 & 56 & - & - & 56 & 115 & 4 & 10 & - & 8 & 44 \\
\hline Gulf & 30 & 2 & - & - & - & - & - & - & - & 12 & - & - & 3 & - & 13 \\
\hline Holmes & 672 & 16 & 16 & 15 & 14 & 167 & - & 4 & 163 & 286 & 18 & 4 & 61 & 11 & 64 \\
\hline Jackson & 920 & 29 & 55 & 40 & 14 & 357 & - & 34 & 323 & 312 & 20 & 22 & 2 & 3 & 66 \\
\hline Jefferson & 418 & 13 & 28 & 53 & 37 & 83 & 2 & 1 & 80 & 109 & 3 & 9 & 2 & 10 & 71 \\
\hline Leon & 281 & 3 & 7 & 21 & 31 & 36 & 2 & - & 34 & 67 & 1 & 10 & 5 & 6 & 94 \\
\hline Liberty & 67 & - & - & 1 & 3 & 1 & - & - & 1 & 24 & 2 & 1 & 4 & 1 & 30 \\
\hline Okaloosa & 465 & 1 & 27 & 23 & 21 & 121 & - & 9 & 112 & 141 & 4 & 10 & 19 & 13 & 85 \\
\hline Santa Rosa & 505 & 7 & 14 & 30 & 29 & 154 & - & 31 & 123 & 141 & 3 & 13 & 2 & 15 & 97 \\
\hline Wakulla & 126 & - & 8 & 2 & 5 & 6 & - & - & 6 & 41 & - & 24 & 6 & 3 & 31 \\
\hline Walton & 540 & 12 & 10 & 46 & 7 & 111 & - & 4 & 107 & 212 & 10 & 8 & 39 & 9 & 76 \\
\hline Washington & 391 & 29 & 9 & 18 & 12 & 104 & - & - & 104 & 157 & 6 & 2 & 9 & 4 & 41 \\
\hline Total & 5,719 & 176 & 219 & 369 & 267 & 1,383 & 4 & 114 & 1,265 & 1,876 & 82 & 128 & 162 & 96 & 961 \\
\hline
\end{tabular}

Source: 2002 Census of Agriculture, Florida state and county data, Volume 1, Geographic area series, Part 9, AC-02-A-9, Issued June 2004, National Statistics Service, US Department of Agriculture. 
Table 2. Farm size distribution, and investment in land \& building, machinery, northwest region of Florida, 2002, and percent change, 1997-2002

\begin{tabular}{|c|c|c|c|c|c|c|c|c|c|c|c|c|c|c|c|c|c|}
\hline \multirow[b]{2}{*}{ County } & \multicolumn{4}{|c|}{ Farms } & \multicolumn{2}{|c|}{$\begin{array}{l}\text { Average } \\
\text { farm size }\end{array}$} & \multicolumn{2}{|c|}{$\begin{array}{l}\text { Farms less than } \\
100 \text { acres }\end{array}$} & \multicolumn{2}{|c|}{$\begin{array}{l}\text { Farms } 100 \text { to } \\
400 \text { acres }\end{array}$} & \multicolumn{2}{|c|}{$\begin{array}{l}\text { Farms } 500 \text { acres } \\
\text { or more }\end{array}$} & \multicolumn{4}{|c|}{ Estimated asset value of land \& buildings } & \multirow{2}{*}{$\begin{array}{c}\text { Estimated asset } \\
\text { value, machinery } \\
\text { \& equipment } \\
\end{array}$} \\
\hline & $\begin{array}{l}\widehat{\mathbf{z}} \\
\mathbf{3} \\
\overline{\mathbf{g}} \\
\stackrel{\Phi}{\mathbf{D}}\end{array}$ & $\begin{array}{l}\text { 우 } \\
\text { ? } \\
\text { a }\end{array}$ & $\begin{array}{l}\text { D } \\
\text { d } \\
\text { d } \\
\text { 象 }\end{array}$ & $\begin{array}{l}\text { ㅇ } \\
\text { o } \\
\text { a }\end{array}$ & 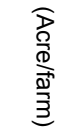 & $\begin{array}{l}\text { ㅇ } \\
\text { o } \\
\text { a }\end{array}$ & 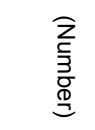 & $\begin{array}{l}\widehat{D} \\
\text { D } \\
\text { D } \\
\text { C. }\end{array}$ & 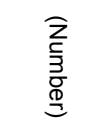 & 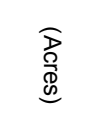 & $\begin{array}{l}\widehat{\underline{z}} \\
\mathbf{3} \\
\overline{\mathbf{0}} \\
\underline{\underline{0}}\end{array}$ & 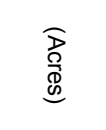 & $\begin{array}{l}\text { बि } \\
\text { ठㅇㅇ }\end{array}$ & $\begin{array}{l}\text { a } \\
\text { ? } \\
\text { a }\end{array}$ & 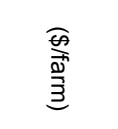 & 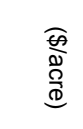 & \\
\hline Bay & 116 & 17 & 10,863 & 29 & 94 & 11 & 103 & 2,643 & 10 & 1,529 & 3 & & 28,244 & 60 & 243,482 & 2,626 & 20,307 \\
\hline Calhoun & 151 & -4 & 49,107 & 11 & 325 & 16 & 72 & 2,718 & 55 & 12,024 & 24 & 34,365 & 72,347 & 31 & 485,551 & 1,596 & 59,619 \\
\hline Escambia & 674 & 10 & 64,581 & 8 & 96 & -1 & 545 & 15,440 & 102 & 20,961 & 27 & 28,180 & 226,444 & 69 & 337,473 & 2,383 & 19,225 \\
\hline Franklin & 20 & -31 & & & & & 17 & 91 & 2 & & 1 & & 7,128 & -8 & 356,396 & 1,165 & 30,118 \\
\hline Gadsden & 343 & -2 & 68,140 & 15 & 199 & 18 & 203 & 7,234 & 119 & 26,071 & 21 & 10,431 & 188,917 & 11 & 550,780 & 2,421 & 38,211 \\
\hline Gulf & 30 & -32 & 4,521 & 25 & 151 & 84 & 17 & 607 & 12 & 2,554 & 1 & & 8,753 & 54 & 301,835 & 1,886 & 14,707 \\
\hline Holmes & 672 & -8 & 90,875 & -5 & 135 & 4 & 397 & 18,950 & 249 & 44,098 & 26 & 19,007 & 149,661 & 7 & 223,042 & 1,610 & 26,081 \\
\hline Jackson & 920 & -4 & 226,890 & -7 & 247 & -2 & 498 & 22,343 & 316 & 67,956 & 106 & 136,591 & 367,887 & 44 & 400,313 & 1,478 & 47,604 \\
\hline Jefferson & 418 & -1 & 132,727 & 2 & 318 & 4 & 274 & 8,158 & 102 & 23,146 & 42 & 101,423 & 244,784 & 40 & 584,211 & 1,850 & 44,245 \\
\hline Leon & 281 & -12 & 74,004 & 5 & 263 & 18 & 218 & 5,639 & 47 & 9,655 & 16 & 58,710 & 148,578 & 16 & 530,636 & 2,085 & 26,624 \\
\hline Liberty & 67 & 6 & 9,900 & 14 & 148 & 7 & 41 & 666 & 23 & 3,465 & 3 & & 13,395 & 6 & 199,919 & 1,366 & 22,610 \\
\hline Okaloosa & 465 & 5 & 55,119 & -2 & 119 & -6 & 326 & 11,731 & 123 & 16,802 & 16 & 13,404 & 136,398 & 39 & 292,073 & 2,539 & 70,199 \\
\hline Santa Rosa & 505 & -9 & 83,790 & -3 & 166 & 7 & 372 & 10,657 & 89 & 19,649 & 44 & 53,484 & 202,917 & 50 & 401,815 & 2,649 & 53,635 \\
\hline Wakulla & 126 & 0 & 10,900 & -8 & 87 & -7 & 95 & 1,973 & 27 & 5,438 & 4 & 1,680 & 26,489 & 22 & 210,229 & 2,891 & 26,466 \\
\hline Walton & 540 & -14 & 79,910 & -8 & 148 & 8 & 356 & 14,916 & 156 & 30,307 & 28 & 34,687 & 176,306 & 51 & 325,288 & 1,889 & 35,615 \\
\hline Washington & 391 & -6 & 53,251 & -11 & 136 & -6 & 236 & 10,218 & 136 & 28,713 & 19 & 14,320 & 129,058 & 51 & 329,229 & 2,288 & 50,772 \\
\hline Total & 5,719 & -4 & $1,014,578$ & -1 & 175 & 7 & 3,770 & 133,984 & 1,568 & 312,368 & 381 & 506,282 & $2,127,306$ & 37 & 360,767 & 2,045 & 36,627 \\
\hline
\end{tabular}

Source: 2002 Census of Agriculture, Florida state and county data, Volume 1, Geographic area series, Part 9, AC-02-A-9, Issued June 2004, National Statistics Service, US Department of Agriculture. 
Table 3A. Farmland Use, northwest region of Florida, 2002, and percent change, 1997-2002

\begin{tabular}{|c|c|c|c|c|c|c|c|c|c|c|}
\hline \multirow{2}{*}{ County } & \multicolumn{2}{|c|}{ All cropland } & \multicolumn{2}{|c|}{ Harvested cropland } & \multicolumn{2}{|c|}{$\begin{array}{l}\text { Cropland used only } \\
\text { for pasture or grazing }\end{array}$} & \multicolumn{2}{|c|}{$\begin{array}{l}\text { Cropland in cultivated } \\
\text { summer fallow }\end{array}$} & \multirow{2}{*}{$\begin{array}{c}\begin{array}{c}\text { Nursery, greenhouse, } \\
\text { floriculture (1), under glass or } \\
\text { other protection }\end{array} \\
\text { (Square feet) }\end{array}$} & \multirow{2}{*}{$\begin{array}{c}\text { Nursery, greenhouse, } \\
\text { floriculture (1), in the open }\end{array}$} \\
\hline & (Acres) & (\% chg) & (Acres & (\% chg) & (Acres) & (\% chg) & (Acres) & (\% chg) & & \\
\hline Bay & 2,976 & -4 & 1,256 & 19 & 1,054 & -1 & & & 25,780 & \\
\hline Calhoun & 24,142 & -11 & 19,186 & -4 & 2,020 & -48 & 142 & & & 702 \\
\hline Escambia & 39,246 & 11 & 28,456 & 0 & 6,268 & 284 & 132 & 128 & 365,098 & 213 \\
\hline \multicolumn{11}{|l|}{ Franklin } \\
\hline Gadsden & 15,253 & -37 & 7,616 & -46 & 3,919 & -3 & 272 & 339 & & 1,110 \\
\hline Gulf & 1,027 & 18 & 114 & -46 & & & & & & \\
\hline Holmes & 36,695 & -8 & 17,694 & -3 & 6,802 & 26 & 945 & 168 & 23,040 & 5 \\
\hline Jackson & 114,428 & -11 & 73,936 & -14 & 18,819 & 5 & 1,455 & -8 & & 57 \\
\hline Jefferson & 29,884 & -10 & 16,347 & -7 & 7,206 & 22 & 354 & & 66,060 & 686 \\
\hline Leon & 11,425 & -31 & 3,705 & -20 & 4,388 & -42 & & & & 289 \\
\hline Liberty & 1,232 & 4 & 223 & -20 & & & - & & & \\
\hline Okaloosa & 19,684 & -6 & 10,739 & 7 & 4,982 & 58 & 36 & -68 & & 116 \\
\hline Santa Rosa & 51,976 & -5 & 45,057 & 1 & 3,036 & -12 & 250 & 0 & 82,794 & 694 \\
\hline Wakulla & 3,055 & -10 & $1,01 \varepsilon$ & -28 & 1,103 & 493 & & & & 16 \\
\hline Walton & 32,143 & -10 & 12,570 & -15 & 10,725 & 34 & 501 & 332 & 6,868 & \\
\hline Washington & 18,404 & -27 & 8,512 & -36 & & & & & & \\
\hline Total & 401,570 & -11 & 246,429 & -10 & 70,322 & 13 & 4,087 & 56 & 569,640 & 3,888 \\
\hline
\end{tabular}

(1) Aquatic Plants, Mushrooms, Flower \& Vegetable seeds and Sod Harvested

Source: 2002 Census of Agriculture, Florida state and county data, Volume 1, Geographic area series, Part 9, AC-02-A-9, Issued June 2004, National Statistics Service, US Department of Agriculture. 
Table 3B. Farmland use, northwest region of Florida, 2002, and percent change, 1997-2002

\begin{tabular}{|c|c|c|c|c|c|c|c|c|c|c|c|c|c|c|}
\hline \multirow{2}{*}{ County } & \multicolumn{2}{|c|}{$\begin{array}{l}\text { Pasture \& rangeland, } \\
\text { other than cropland } \\
\& \text { woodland pastured }\end{array}$} & \multicolumn{2}{|c|}{$\begin{array}{l}\text { Pastureland, } \\
\text { all types }\end{array}$} & \multicolumn{2}{|c|}{ Woodland } & \multicolumn{2}{|c|}{$\begin{array}{l}\text { Woodland } \\
\text { pastured }\end{array}$} & \multicolumn{2}{|c|}{$\begin{array}{l}\text { Woodland } \\
\text { not pastured }\end{array}$} & \multicolumn{2}{|c|}{$\begin{array}{l}\text { Land in conservation } \\
\text { or wetlands reserve } \\
\text { programs }\end{array}$} & \multirow{2}{*}{ 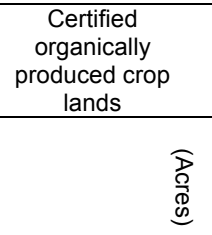 } & \multirow{2}{*}{$\begin{array}{l}\text { Land enrolled in } \\
\text { federal or other } \\
\text { crop insurance } \\
\text { programs } \\
\end{array}$} \\
\hline & 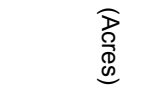 & $\begin{array}{l}\text { बे } \\
\text { के } \\
\text { a }\end{array}$ & 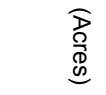 & $\begin{array}{l}\text { o } \\
\text { 高 } \\
\text { a्d }\end{array}$ & 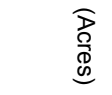 & $\begin{array}{l}\text { o } \\
\text { 产 } \\
\text { a }\end{array}$ & 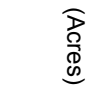 & $\begin{array}{l}\text { ò } \\
\text { 高 }\end{array}$ & 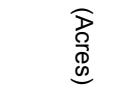 & $\begin{array}{l}\text { o } \\
\text { 高 } \\
\text { a्d }\end{array}$ & 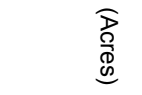 & $\begin{array}{l}\text { o } \\
\text { 高 } \\
\text { a }\end{array}$ & & \\
\hline Bay & 1,309 & -10 & 2,910 & -5 & 5,394 & 63 & 935 & 39 & 4,459 & 69 & & & & \\
\hline Calhoun & 3,642 & 196 & 7,698 & 43 & 19,412 & 36 & 1,120 & 25 & 18,292 & 37 & 1,990 & -59 & & 14,996 \\
\hline Escambia & 6,254 & 118 & 14,290 & 9 & 14,565 & -6 & 3,514 & -27 & 11,051 & 3 & 3,077 & -2 & & 22,457 \\
\hline Franklin & & & & & 668 & -83 & & & & & & & & \\
\hline Gadsden & 5,117 & 40 & 14,752 & 11 & 43,511 & 73 & 5,917 & 59 & 37,594 & 76 & 2,196 & -22 & 21 & 880 \\
\hline \multicolumn{15}{|l|}{ Gulf } \\
\hline Holmes & 15,682 & 95 & 35,578 & 10 & 29,034 & -27 & 7,697 & -6 & 21,337 & -33 & 8,818 & -29 & & 8,706 \\
\hline Jackson & 38,481 & 62 & 71,406 & 19 & 61,904 & -18 & 11,252 & -2 & 50,652 & -21 & 17,508 & 3 & 257 & 47,150 \\
\hline Jackson & 38,481 & 62 & 71,406 & 19 & 61, & & & & & & & & & \\
\hline Jefferson & 10,462 & -29 & 31,352 & -37 & 78,797 & 7 & 14,559 & -43 & 64,238 & 33 & 7,603 & 10 & 39 & 5,449 \\
\hline Leon & 5,788 & 5 & 11,320 & -1 & 51,455 & 29 & 2,200 & 37 & 49,255 & 29 & 1,310 & -80 & 36 & \\
\hline Liberty & & & 6,676 & 22 & 7,042 & 7 & 4,279 & 0 & 2,763 & 21 & & & & \\
\hline Okaloosa & 5,333 & 14 & 20,019 & 7 & 25,349 & -7 & 10,723 & 65 & 14,626 & -30 & 6,333 & 3 & & 5,510 \\
\hline Santa Rosa & 4,634 & -26 & 18,953 & -5 & 22,676 & 8 & 10,436 & 49 & 12,240 & -12 & 4,548 & 18 & & 34,949 \\
\hline Wakulla & 1,268 & -20 & 4,457 & -16 & 5,935 & 2 & 2,255 & 16 & 3,680 & -5 & & & 6 & \\
\hline Walton & 16,861 & 47 & 30,902 & -2 & 25,387 & -23 & 5,193 & -27 & 20,194 & -22 & 6,097 & -48 & & 3,801 \\
\hline \multicolumn{15}{|l|}{ Washington } \\
\hline Total & 114,831 & 34 & 270,313 & 0 & 391,129 & 2 & 80,080 & -4 & 310,381 & 5 & 59,480 & -21 & 359 & 143,898 \\
\hline
\end{tabular}

Source: 2002 Census of Agriculture, Florida state and county data, Volume 1, Geographic area series, Part 9, AC-02-A-9, Issued June 2004, National Statistics Service, US Department of Agriculture 
Table 4. Market value of agricultural products sold, value of government payments, production expenses, and net farm cash income from operation, northwest region of Florida, 2002,and percent change, 1997-2002

\begin{tabular}{|c|c|c|c|c|c|c|c|c|c|}
\hline \multirow{2}{*}{ County } & \multicolumn{4}{|c|}{ Market value of all agricultural products sold } & \multirow{2}{*}{$\begin{array}{c}\begin{array}{c}\text { Value of government } \\
\text { payments }\end{array} \\
(\$ 1000)\end{array}$} & \multicolumn{2}{|c|}{ Total farm production expenses } & \multicolumn{2}{|c|}{ Net farm cash income from operation } \\
\hline & $(\$ 1000)$ & (\% chg) & (\$/farm) & (\% chg) & & $(\$ 1000)$ & (\$/farm) & $(\$ 1000)$ & $(\$ /$ farm $)$ \\
\hline Bay & 2,157 & -23 & 18,592 & -34 & & 1,911 & 16,476 & 1,206 & 10,398 \\
\hline Calhoun & 14,390 & -12 & 95,300 & -9 & 1,046 & 14,312 & 96,055 & 2,441 & 16,383 \\
\hline Escambia & 15,676 & -5 & 23,258 & -14 & 2,473 & 17,062 & 25,428 & -875 & $-1,305$ \\
\hline Franklin & 385 & & 19,257 & & & 199 & 9,940 & 191 & 9,542 \\
\hline Gadsden & 91,377 & -2 & 266,404 & 1 & 148 & 62,592 & 182,483 & 29,881 & 87,117 \\
\hline Gulf & 489 & 24 & 16,308 & 83 & & 523 & 18,035 & 72 & 2,491 \\
\hline Holmes & 30,223 & -22 & 44,975 & -14 & 908 & 24,726 & 36,850 & 7,734 & 11,526 \\
\hline Jackson & 36,470 & -28 & 39,641 & -25 & 3,158 & 46,483 & 50,581 & $-6,214$ & $-6,762$ \\
\hline Jefferson & 21,154 & 15 & 50,608 & 16 & 606 & 26,189 & 62,503 & -294 & -701 \\
\hline Leon & 6,659 & 78 & 23,699 & 101 & 201 & 8,797 & 31,418 & -175 & -625 \\
\hline Liberty & 1,497 & 123 & 22,339 & 110 & & 925 & 13,800 & 401 & 5,982 \\
\hline Okaloosa & 6,540 & -33 & 14,065 & -36 & 418 & 8,423 & 18,036 & $-1,220$ & $-2,613$ \\
\hline Santa Rosa & 20,997 & -28 & 41,579 & -21 & 2,423 & 21,602 & 42,776 & 1,015 & 2,010 \\
\hline Wakulla & 1,584 & -54 & 12,571 & -54 & 34 & 1,755 & 13,930 & -101 & -801 \\
\hline Walton & 20,055 & -16 & 37,139 & -1 & 346 & 27,001 & 49,817 & $-3,183$ & $-5,873$ \\
\hline Washington & 5,506 & -46 & 14,081 & -42 & 411 & 6,112 & 15,591 & 698 & 1,782 \\
\hline Total & 275,159 & -2 & 46,239 & 4 & 12,172 & 268,612 & 42,732 & 31,577 & 8,034 \\
\hline
\end{tabular}

Source: 2002 Census of Agriculture, Florida state and county data, Volume 1, Geographic area series, Part 9, AC-02-A-9, Issued June 2004, National Statistics Service, US Department of Agriculture 
Table 5. Value of agricultural products by commodity group, northwest region of Florida, 2002, and percent change, 1997-2002

\begin{tabular}{|c|c|c|c|c|c|c|c|c|c|c|c|c|c|c|c|c|c|c|c|c|}
\hline \multirow{2}{*}{ County } & \multicolumn{2}{|c|}{$\begin{array}{l}\text { All crops, } \\
\text { Including } \\
\text { nursery and } \\
\text { greenhouse }\end{array}$} & \multirow{2}{*}{ 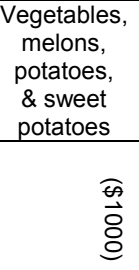 } & \multicolumn{2}{|c|}{$\begin{array}{l}\text { Fruits, tree } \\
\text { nuts \& } \\
\text { berries }\end{array}$} & \multirow{2}{*}{ 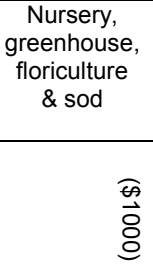 } & \multirow{2}{*}{ 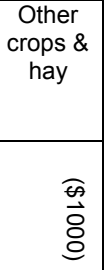 } & \multicolumn{2}{|c|}{$\begin{array}{l}\text { Livestock, } \\
\text { poultry \& their } \\
\text { products }\end{array}$} & \multicolumn{2}{|c|}{ Poultry \& eggs } & \multicolumn{2}{|c|}{ Cattle \& calves } & \multicolumn{2}{|c|}{$\begin{array}{l}\text { Milk \& other } \\
\text { dairy products } \\
\text { from cows }\end{array}$} & \multicolumn{2}{|c|}{ Hogs \& pigs } & \multirow{2}{*}{$\begin{array}{r}\begin{array}{c}\text { Sheep, } \\
\text { goats } \\
\text { \& their } \\
\text { products }\end{array} \\
\qquad \begin{array}{l}\widehat{\oplus} \\
\vec{\circ}\end{array}\end{array}$} & \multirow{2}{*}{ 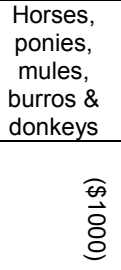 } & \multirow{2}{*}{$\begin{array}{r}\text { Aquaculture } \\
\widehat{\widehat{\theta}} \\
\vec{\circ} \\
\text { }\end{array}$} \\
\hline & $\begin{array}{l}\widehat{\vec{\theta}} \\
\overrightarrow{\bar{O}} \\
\dot{0}\end{array}$ & $\begin{array}{l}\text { o } \\
\text { ô } \\
\text { âb }\end{array}$ & & 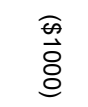 & 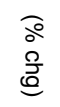 & & & $\begin{array}{l}\text { बि } \\
\text { ठे } \\
\text { ठㅇ }\end{array}$ & $\begin{array}{l}\text { o } \\
\text { 高 } \\
\text { ấ }\end{array}$ & 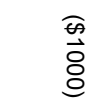 & $\begin{array}{l}\text { oे } \\
\text { के } \\
\text { a }\end{array}$ & $\begin{array}{l}\bar{\theta} \\
\text { ठे } \\
\text { ठㅇ }\end{array}$ & $\begin{array}{l}\text { ०े } \\
\text { 今े } \\
\text { a्d }\end{array}$ & $\begin{array}{l}\widehat{\oplus} \\
\vec{\partial} \\
\text { }\end{array}$ & $\begin{array}{l}\text { o } \\
\text { 官 } \\
\text { a }\end{array}$ & $\begin{array}{l}\hat{\theta} \\
\text { के } \\
\text { ठ }\end{array}$ & $\begin{array}{l}\text { o } \\
\text { 家 } \\
\text { a }\end{array}$ & & & \\
\hline Bay & 1,881 & -24 & 31 & 153 & 89 & 1,527 & 153 & 275 & -15 & 8 & & 56 & -27 & & & 3 & -84 & 1 & 68 & 3 \\
\hline Calhoun & 13,152 & -10 & 161 & & & 7,775 & 2,099 & 1,238 & -30 & & & 546 & 4 & & & 76 & & 4 & 30 & \\
\hline Escambia & 10,370 & 9 & 170 & 617 & & 3,978 & 1,155 & 5,306 & -25 & 4 & & 1,409 & -14 & 2,273 & -41 & 22 & & 14 & 233 & 1,157 \\
\hline Franklin & & & & & & & & 385 & & & & & & & & & & & & \\
\hline Gadsden & 90,268 & 5 & & & & & 140 & 1,108 & -85 & & & 877 & -17 & 118 & & 66 & -62 & & 14 & \\
\hline Gulf & & & & & & & & & & 1 & & 83 & -16 & & & & & & & \\
\hline Holmes & 2,920 & -42 & 925 & 137 & & & 1,244 & 27,303 & -18 & 20,750 & -26 & 3,616 & 42 & 2,632 & -1 & 86 & -49 & & 175 & \\
\hline Jackson & 24,845 & -34 & 6,034 & 479 & & & 10,471 & 11,624 & -12 & & & 5,954 & -1 & 4,568 & -11 & & & 28 & 201 & \\
\hline Jefferson & 9,317 & -35 & 331 & 888 & & 5,086 & 998 & 11,837 & 187 & 9 & & 2,126 & -9 & 9,590 & & 21 & -69 & 14 & 70 & \\
\hline Leon & 4,432 & 210 & 189 & 332 & & 3,262 & 256 & 2,228 & -4 & 3 & -82 & & & & & 34 & -62 & 4 & 137 & 14 \\
\hline Liberty & & & & & & & 19 & & & 8 & & 219 & 89 & & & & & & & \\
\hline Okaloosa & 3,575 & -34 & 168 & 361 & 622 & 1,060 & 1,175 & 2,965 & -31 & 1,845 & -33 & 591 & -40 & & & 26 & -70 & 10 & 180 & \\
\hline Santa Rosa & 19,149 & -29 & 288 & & & 4,842 & 7,229 & 1,848 & -12 & & & 1,269 & -10 & & & 65 & 5 & 44 & 212 & \\
\hline Wakulla & 642 & 26 & & & & 355 & 132 & 942 & -68 & 6 & & 272 & -39 & & & & & & 12 & \\
\hline Walton & 4,538 & -5 & 317 & 739 & & 2,247 & 808 & 15,517 & -18 & 12,452 & -18 & 2,662 & -21 & 180 & & 21 & -58 & & 131 & \\
\hline Washington & 2,288 & -40 & 582 & 341 & 267 & 170 & 672 & 3,217 & -49 & 1,025 & -57 & 1,055 & -47 & 1,028 & -24 & & & & 70 & \\
\hline Total & 187,377 & 0 & 9,196 & 4,047 & 377 & 30,302 & 26,551 & 85,793 & -14 & 36,111 & -43 & 20,735 & -8 & 20,389 & -19 & 420 & -56 & 119 & 1,533 & 1,174 \\
\hline
\end{tabular}

Source: 2002 Census of Agriculture, Florida state and county data, Volume 1, Geographic area series, Part 9, AC-02-A-9, Issued June 2004, National Statistics Service, US Department of Agriculture 
Table 6. Characteristics of principal operator, and primary occupation of farmers, northwest region of Florida, 2002, and percent change, 1997-2002

\begin{tabular}{|c|c|c|c|c|c|c|c|c|c|c|}
\hline \multirow{2}{*}{ County } & \multirow{2}{*}{\begin{tabular}{r|} 
Total operators \\
(Number)
\end{tabular}} & \multirow{2}{*}{$\begin{array}{r}\text { Total women operators } \\
\text { (Number) }\end{array}$} & \multicolumn{2}{|c|}{ Primary occupation farming } & \multicolumn{2}{|c|}{ Primary occupation other } & \multicolumn{4}{|c|}{ Female farms } \\
\hline & & & (Number) & (\% chg) & (Number) & (\% chg) & (Number) & (\% chg) & (Acres) & (\% chg) \\
\hline Bay & 149 & 44 & 69 & 86 & 47 & -24 & 24 & 41 & 343 & -32 \\
\hline Calhoun & 213 & 59 & 75 & -9 & 76 & 1 & 20 & 100 & 2,673 & -59 \\
\hline Escambia & 917 & 263 & 364 & 59 & 310 & -19 & 100 & 59 & 4,505 & -29 \\
\hline Franklin & 34 & 11 & 17 & 31 & 3 & -81 & & & & \\
\hline Gadsden & 499 & 138 & 194 & 46 & 149 & -32 & 37 & -8 & 5,308 & 5 \\
\hline Gulf & 42 & 12 & 10 & -33 & 20 & -31 & 1 & -75 & & \\
\hline Holmes & 889 & 221 & 344 & 13 & 328 & -24 & 68 & -8 & 7,138 & -22 \\
\hline Jackson & 1,194 & 310 & 473 & 2 & 447 & -10 & 121 & 13 & 17,510 & -7 \\
\hline Jefferson & 582 & 189 & 147 & 11 & 271 & -7 & 89 & 107 & 21,308 & 208 \\
\hline Leon & 424 & 156 & 137 & 65 & 144 & -39 & 67 & 56 & 13,769 & -37 \\
\hline Liberty & 82 & 17 & 30 & 30 & 37 & -8 & 10 & -29 & 1,656 & 2 \\
\hline Okaloosa & 704 & 237 & 216 & 44 & 249 & -15 & 67 & 6 & 7,159 & -19 \\
\hline Santa Rosa & 703 & 205 & 265 & 5 & 240 & -22 & 68 & 51 & 3,442 & 3 \\
\hline Wakulla & 180 & 53 & 61 & 24 & 65 & -16 & 23 & -15 & 929 & 7 \\
\hline Walton & 792 & 263 & 260 & 13 & 280 & -30 & 96 & 16 & 7,468 & -21 \\
\hline Washington & 512 & 154 & 187 & 26 & 204 & -24 & 71 & 69 & 5,764 & 9 \\
\hline Total & 7,916 & 2,332 & 2,849 & 21 & 2,870 & -21 & 862 & 28 & 98,972 & -5 \\
\hline
\end{tabular}

Source: 2002 Census of Agriculture, Florida state and county data, Volume 1, Geographic area series, Part 9, AC-02-A-9, Issued June 2004, National Statistics Service, US Department of Agriculture 
Table 7. Type of business organization, northwest region of Florida, 2002, and percent change, 1997-2002

\begin{tabular}{|c|c|c|c|c|c|c|c|c|c|c|c|c|c|c|c|c|c|}
\hline \multirow[b]{2}{*}{ County } & \multicolumn{4}{|c|}{ Family or individual farms } & \multicolumn{4}{|c|}{ Partnership farms } & \multicolumn{4}{|c|}{ Corporation family-held farms } & \multicolumn{2}{|c|}{$\begin{array}{l}\text { Corporation other than } \\
\text { family held farms }\end{array}$} & \multicolumn{3}{|c|}{$\begin{array}{l}\text { Other-cooperative, estate } \\
\text { or trust, institutional, etc }\end{array}$} \\
\hline & 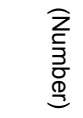 & $\begin{array}{l}\widehat{\circ} \\
\text { a } \\
\text { a }\end{array}$ & 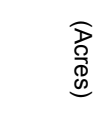 & $\begin{array}{l}\text { 우 } \\
\text { 产 }\end{array}$ & 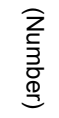 & 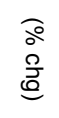 & $\begin{array}{l}\widehat{P} \\
\text { 员 } \\
\text { 禹 }\end{array}$ & $\begin{array}{l}\widehat{\circ} \\
\text { o } \\
\text { a }\end{array}$ & 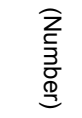 & $\begin{array}{l}\text { a } \\
\text { o } \\
\text { a }\end{array}$ & 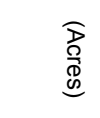 & $\begin{array}{l}\text { 우 } \\
\text { 产 }\end{array}$ & 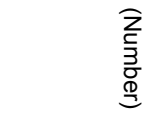 & $\begin{array}{l}\widehat{\circ} \\
\text { 高 } \\
\text { a }\end{array}$ & 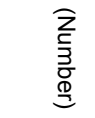 & $\begin{array}{l}\text { o } \\
\text { a } \\
\text { â }\end{array}$ & 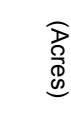 \\
\hline Bay & 105 & 19 & 5,103 & -17 & 7 & 75 & & & 3 & -57 & & & 1 & & & & \\
\hline Calhoun & 135 & -3 & 38,185 & 42 & 4 & -43 & 8,483 & -4 & 6 & -40 & 458 & & 1 & & 5 & 400 & \\
\hline Escambia & 650 & 15 & 59,104 & 24 & 15 & -50 & & & 6 & -33 & 72 & -94 & 1 & -75 & 2 & -60 & \\
\hline Franklin & 13 & -38 & 611 & -85 & 6 & -25 & 6 & & 1 & & & & & & & & \\
\hline Gadsden & 304 & 0 & 52,744 & 37 & 24 & 14 & 6,790 & -22 & 11 & -42 & 4,820 & -44 & 2 & -67 & 2 & 100 & \\
\hline Gulf & 26 & -30 & 3,791 & 27 & 4 & 100 & 730 & & & & & & & & & & \\
\hline Holmes & 644 & -8 & 81,113 & -6 & 22 & -12 & 7,189 & 24 & 5 & -17 & & & & & 1 & -83 & \\
\hline Jackson & 856 & 0 & 186,616 & -2 & 38 & -47 & 25,113 & -8 & 19 & -37 & 8,627 & -61 & & & 7 & 133 & 6,534 \\
\hline Jefferson & 370 & -1 & & & 28 & 22 & 56,677 & 230 & 18 & -14 & 14,801 & 154 & 2 & 100 & & & \\
\hline Leon & 239 & -16 & & & 24 & 33 & 19,706 & 27 & 17 & 89 & 34,440 & & & & 1 & -67 & \\
\hline Liberty & 62 & 24 & 9,657 & 75 & 4 & -33 & & & 1 & -86 & & & & & & & \\
\hline Okaloosa & 450 & 11 & 45,740 & -2 & 6 & -79 & & & 9 & 29 & & & & & & & \\
\hline Santa Rosa & 475 & -6 & 68,741 & -3 & 16 & -30 & 7,762 & -1 & 9 & -44 & & & 3 & -40 & 2 & -67 & \\
\hline Wakulla & 123 & 7 & & & 3 & -50 & & & & & & & & & & & \\
\hline Walton & 511 & -14 & & & 20 & -23 & 9,559 & 92 & 6 & -40 & 2,772 & -51 & 1 & 0 & 2 & -33 & \\
\hline Washington & 383 & -1 & 50,675 & -6 & 4 & -69 & 1,756 & -22 & 3 & -70 & & & & & 1 & -83 & \\
\hline Total & 5,346 & -1 & 602,080 & -24 & 225 & -28 & 143,771 & 25 & 114 & -33 & 65,990 & 25 & 11 & -59 & 23 & -43 & 6,534 \\
\hline
\end{tabular}

Source: 2002 Census of Agriculture, Florida state and county data, Volume 1, Geographic area series, Part 9, AC-02-A-9, Issued June 2004, National Statistics Service, US Department of Agriculture 
Table 8. Ethnicity of farm operators, northwest region of Florida, 2002

\begin{tabular}{|c|c|c|c|c|c|c|c|c|c|c|c|c|}
\hline \multirow{2}{*}{ County } & \multicolumn{3}{|c|}{ White } & \multicolumn{3}{|c|}{ Black or African American } & \multicolumn{3}{|c|}{ Spanish, Hispanic, or Latino oOrigin } & \multicolumn{3}{|c|}{ Other ethnic groups* } \\
\hline & (Farms) & (Operators) & (Acres) & (Farms) & (Operators) & (Acres) & (Farms) & (Operators) & (Acres) & (Farms) & (Operators) & (Acres) \\
\hline Bay & 109 & 138 & 10723 & 3 & 3 & 120 & 1 & 1 & & 7 & 8 & 20 \\
\hline Calhoun & 146 & 193 & 48897 & 5 & 5 & 210 & 4 & 4 & 68 & 4 & 6 & 160 \\
\hline Escambia & 617 & 822 & 63268 & 27 & 47 & 526 & 12 & 12 & 471 & 38 & 38 & 1105 \\
\hline Franklin & 20 & 34 & & & & & & & & 0 & 0 & 0 \\
\hline Gadsden & 307 & 436 & 65878 & 44 & 54 & 2584 & 6 & 6 & 791 & 2 & 2 & 0 \\
\hline Gulf & 30 & 42 & 4521 & & & & & & & 0 & 0 & 0 \\
\hline Holmes & 653 & 854 & 88950 & 14 & 14 & 1268 & 8 & 8 & 276 & 11 & 15 & 851 \\
\hline Jackson & 832 & 1066 & 213746 & 87 & 104 & 15193 & 11 & 11 & 671 & 14 & 14 & 1300 \\
\hline Jefferson & 379 & 534 & 131571 & 37 & 40 & 1637 & 16 & 21 & 3575 & 6 & 6 & 129 \\
\hline Leon & 238 & 341 & 73017 & 46 & 61 & 949 & 8 & 8 & 292 & 8 & 8 & 100 \\
\hline Liberty & 67 & 82 & 9900 & & & & & & & 0 & 0 & 0 \\
\hline Okaloosa & 461 & 678 & 54829 & & & & 9 & 13 & 722 & 12 & 15 & 498 \\
\hline Santa Rosa & 482 & 649 & 83282 & 9 & 9 & 65 & 5 & 7 & 556 & 26 & 39 & 833 \\
\hline Wakulla & 107 & 160 & 10759 & 19 & 19 & 141 & 10 & 12 & 275 & 0 & 0 & 0 \\
\hline Walton & 530 & 739 & 78690 & 3 & 3 & 338 & 15 & 20 & 1432 & 23 & 26 & 2192 \\
\hline Washington & 369 & 474 & 51749 & 5 & 5 & 640 & 3 & 3 & 250 & 30 & 32 & 3610 \\
\hline Total & 5,347 & 7,242 & 989,780 & 299 & 364 & 23,671 & 108 & 126 & 9,379 & 181 & 209 & 10,798 \\
\hline
\end{tabular}

Source: 2002 Census of Agriculture, Florida state and county data, Volume 1, Geographic area series, Part 9, AC-02-A-9, Issued June 2004, National Statistics Service, US Department of Agriculture 
Table 9. Output impacts of agriculture and natural resource industries, northwest region of Florida, 2002 data

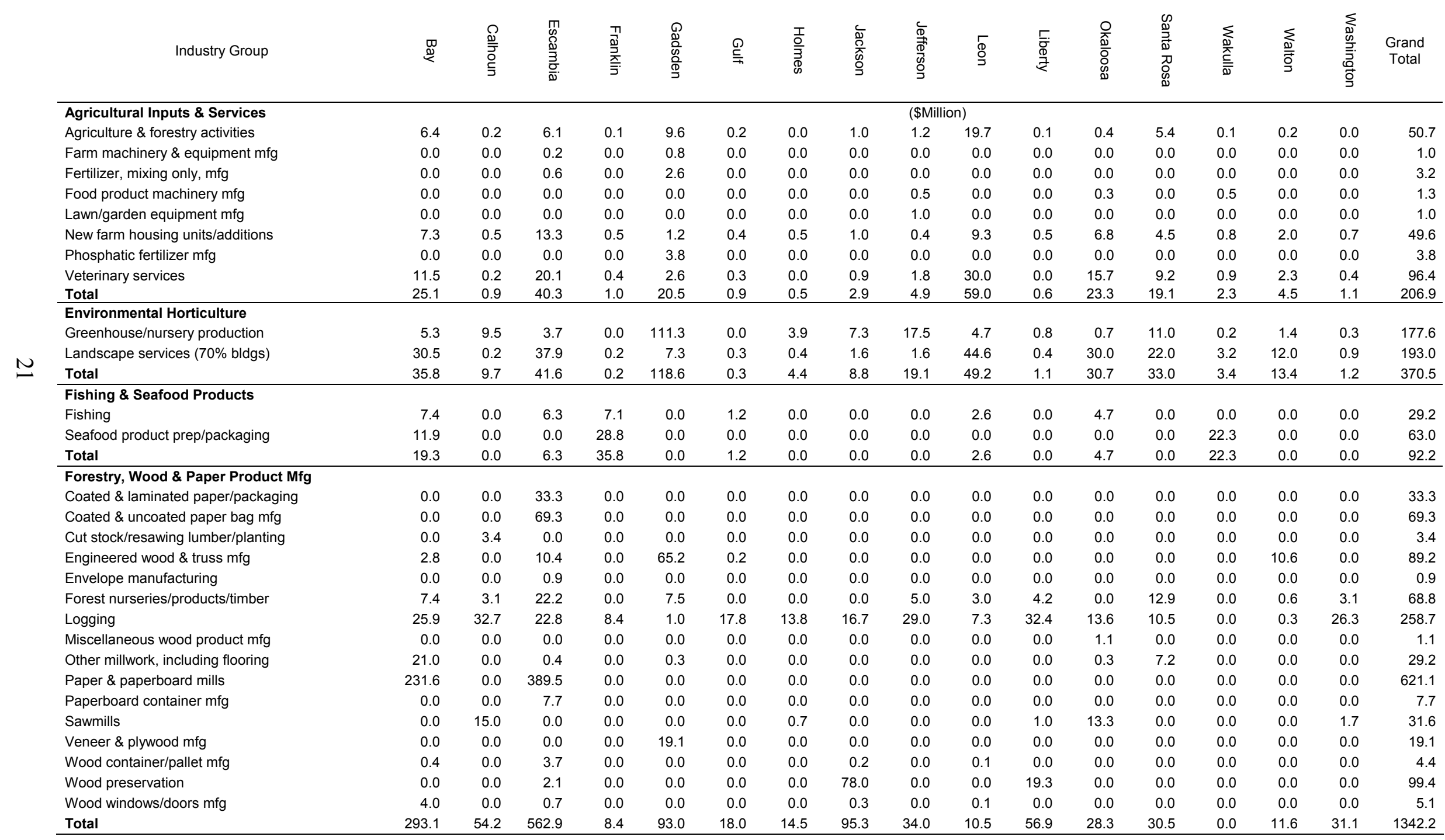


Table 9 (continued). Output impacts of agriculture and natural resource industries, northwest region of Florida, 2002 data

\begin{tabular}{|c|c|c|c|c|c|c|c|c|c|c|c|c|c|c|c|c|c|}
\hline Industry Group & $\stackrel{\text { W }}{\stackrel{\mathscr{N}}{2}}$ & 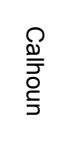 & 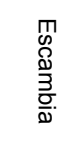 & 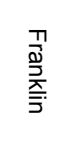 & $\begin{array}{l}\mathbb{Q} \\
\mathscr{D} \\
0 \\
0 \\
\mathbb{Q} \\
\mathcal{O}\end{array}$ & $\stackrel{\oplus}{\rightleftharpoons}$ & 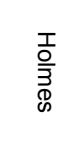 & 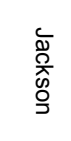 & 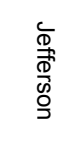 & $\begin{array}{l}\text { ర్ } \\
\text { อ }\end{array}$ & $\begin{array}{l}\frac{\Gamma}{\bar{\sigma}} \\
\stackrel{\Phi}{\gtrless} \\
\stackrel{2}{2}\end{array}$ & 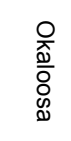 & $\begin{array}{l}\infty \\
\mathscr{D} \\
\stackrel{\Xi}{\vec{D}} \\
\mathbb{D} \\
D \\
0 \\
\mathbb{D}\end{array}$ & 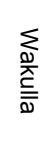 & 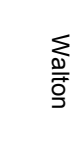 & 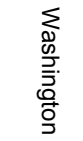 & $\begin{array}{l}\text { Grand } \\
\text { Total }\end{array}$ \\
\hline Fruit \& Vegetable Farming \& & & \multicolumn{16}{|c|}{ (\$Million) } \\
\hline Frozen food manufacturing & 1.3 & 0.0 & 0.0 & 0.0 & 0.0 & 0.0 & 0.0 & 0.0 & 0.0 & 0.0 & 0.0 & 0.0 & 0.0 & 0.0 & 0.0 & 0.0 & 1.3 \\
\hline Fruit farming & 0.9 & 0.1 & 3.7 & 0.0 & 6.1 & 0.0 & 1.6 & 4.3 & 4.6 & 2.3 & 0.0 & 1.8 & 1.5 & 0.0 & 1.7 & 5.2 & 34.0 \\
\hline Vegetable \& melon farming & 0.3 & 0.4 & 0.4 & 0.0 & 79.4 & 0.0 & 3.0 & 48.3 & 1.2 & 0.5 & 0.0 & 0.4 & 1.5 & 6.9 & 0.6 & 3.3 & 146.2 \\
\hline Total & 2.4 & 0.5 & 4.2 & 0.0 & 85.5 & 0.0 & 4.6 & 52.7 & 5.8 & 2.8 & 0.0 & 2.1 & 3.0 & 6.9 & 2.3 & 8.5 & 181.4 \\
\hline \multicolumn{18}{|l|}{ Grain \& Oilseed Farming \& Processing } \\
\hline Flour milling & 0.0 & 0.0 & 0.0 & 0.0 & 0.0 & 0.0 & 0.0 & 0.0 & 0.0 & 0.0 & 0.0 & 0.0 & 1.8 & 0.0 & 0.0 & 0.0 & 1.8 \\
\hline Grain farming & 0.2 & 0.1 & 1.1 & 0.0 & 0.3 & 0.0 & 0.2 & 4.0 & 1.8 & 0.2 & 0.0 & 0.1 & 0.2 & 0.0 & 0.1 & 0.6 & 8.9 \\
\hline Oilseed farming & 0.0 & 0.1 & 0.6 & 0.0 & 0.0 & 0.0 & 0.1 & 0.6 & 0.2 & 0.0 & 0.0 & 0.0 & 0.4 & 0.0 & 0.0 & 0.3 & 2.4 \\
\hline Total & 0.2 & 0.3 & 1.7 & 0.0 & 0.3 & 0.0 & 0.3 & 4.5 & 2.0 & 0.2 & 0.0 & 0.1 & 2.4 & 0.0 & 0.1 & 1.0 & 13.1 \\
\hline \multicolumn{18}{|c|}{ Livestock \& Dairy Farming \& Animal Products Mfg } \\
\hline Animal production, except cattle \& poultry & 1.3 & 1.1 & 3.7 & 0.0 & 4.3 & 0.0 & 1.0 & 4.2 & 0.3 & 6.8 & 1.4 & 6.9 & 3.7 & 1.9 & 8.5 & 0.8 & 45.8 \\
\hline Animal, except poultry, slaughtering & 0.0 & 0.0 & 9.2 & 0.0 & 7.6 & 0.0 & 0.0 & 0.0 & 0.0 & 0.0 & 0.0 & 0.0 & 0.0 & 0.0 & 0.0 & 6.3 & 23.1 \\
\hline Cattle ranching \& farming & 0.3 & 2.9 & 10.9 & 0.0 & 3.8 & 0.0 & 18.5 & 36.7 & 21.0 & 4.7 & 0.3 & 2.4 & 3.4 & 0.1 & 6.3 & 15.6 & 126.7 \\
\hline Dry/condensed/evaporated dairy products & 0.0 & 0.0 & 0.0 & 0.0 & 0.0 & 0.0 & 0.0 & 0.0 & 0.0 & 0.0 & 0.0 & 0.3 & 0.0 & 0.0 & 0.0 & 0.0 & 0.3 \\
\hline Ice cream \& frozen dessert mfg & 0.0 & 0.0 & 0.5 & 0.0 & 0.0 & 0.0 & 0.0 & 0.0 & 0.0 & 0.0 & 0.0 & 0.0 & 0.0 & 0.0 & 0.0 & 0.0 & 0.5 \\
\hline Meat processed from carcasses & 0.0 & 0.0 & 0.3 & 0.0 & 0.0 & 0.0 & 0.0 & 0.0 & 0.0 & 0.0 & 0.0 & 0.0 & 0.0 & 0.0 & 0.0 & 0.0 & 0.3 \\
\hline Poultry \& egg production & 0.0 & 0.4 & 0.3 & 0.0 & 0.6 & 0.0 & 45.9 & 3.3 & 0.5 & 0.0 & 0.0 & 3.4 & 0.0 & 0.0 & 19.7 & 1.1 & 75.1 \\
\hline Poultry processing & 0.0 & 0.0 & 0.0 & 0.0 & 0.0 & 0.0 & 0.0 & 0.0 & 0.0 & 0.0 & 0.0 & 0.0 & 0.0 & 0.0 & 135.9 & 0.0 & 135.9 \\
\hline Total & 1.5 & 4.4 & 24.9 & 0.0 & 16.3 & 0.0 & 65.4 & 44.1 & 21.8 & 11.4 & 1.7 & 13.0 & 7.1 & 2.0 & 170.4 & 23.8 & 407.8 \\
\hline \multicolumn{18}{|l|}{ Mining } \\
\hline Drilling oil \& gas wells & 0.0 & 0.0 & 0.2 & 0.0 & 0.0 & 0.0 & 0.0 & 0.4 & 0.0 & 0.0 & 0.0 & 0.0 & 0.5 & 0.0 & 0.0 & 0.0 & 1.0 \\
\hline Oil \& gas extraction & 0.7 & 0.0 & 3.9 & 0.0 & 0.0 & 0.0 & 0.0 & 0.0 & 0.0 & 0.0 & 0.0 & 0.0 & 166.0 & 0.0 & 0.0 & 0.0 & 170.6 \\
\hline Other nonmetallic mineral mining & 0.0 & 0.0 & 0.0 & 0.0 & 0.0 & 0.0 & 0.0 & 0.0 & 0.0 & 0.0 & 0.0 & 0.0 & 0.5 & 0.0 & 0.0 & 0.0 & 0.5 \\
\hline Sand/gravel/clay/refractory mining & 0.0 & 0.0 & 11.2 & 0.0 & 26.0 & 0.0 & 0.0 & 0.0 & 0.0 & 0.4 & 0.0 & 0.6 & 1.7 & 0.0 & 0.7 & 0.0 & 40.6 \\
\hline Stone mining \& quarrying & 6.1 & 0.0 & 1.2 & 0.0 & 0.0 & 0.0 & 0.0 & 9.6 & 0.0 & 0.0 & 0.0 & 0.0 & 0.0 & 0.0 & 0.0 & 0.0 & 17.0 \\
\hline Support activities for oil \& gas & 0.0 & 0.0 & 0.2 & 0.0 & 0.0 & 0.0 & 0.0 & 0.0 & 0.0 & 0.2 & 0.0 & 0.0 & 0.5 & 0.0 & 0.0 & 0.0 & 0.9 \\
\hline Total & 6.8 & 0.0 & 16.6 & 0.0 & 26.0 & 0.0 & 0.0 & 10.0 & 0.0 & 0.5 & 0.0 & 0.6 & 169.2 & 0.0 & 0.7 & 0.0 & 230.5 \\
\hline \multicolumn{18}{|l|}{ Other Crop Farming } \\
\hline All other crop farming & 0.4 & 2.1 & 2.6 & 0.0 & 1.0 & 0.0 & 1.8 & 21.5 & 1.3 & 0.3 & 0.0 & 1.4 & 14.0 & 0.0 & 1.9 & 1.8 & 50.1 \\
\hline Cotton farming & 0.0 & 2.2 & 6.0 & 0.0 & 0.0 & 0.0 & 0.6 & 11.2 & 0.4 & 0.0 & 0.0 & 1.1 & 11.8 & 0.0 & 0.4 & 0.3 & 34.1 \\
\hline Tree nut farming & 0.0 & 0.0 & 0.3 & 0.0 & 0.0 & 0.0 & 0.0 & 0.2 & 0.3 & 0.0 & 0.0 & 0.0 & 0.0 & 0.0 & 0.0 & 0.1 & 0.9 \\
\hline Total & 0.4 & 4.3 & 8.9 & 0.0 & 1.0 & 0.0 & 2.5 & 33.0 & 2.0 & 0.3 & 0.0 & 2.5 & 25.8 & 0.0 & 2.3 & 2.2 & 85.2 \\
\hline
\end{tabular}


Table 9 (continued). Output impacts of agriculture and natural resource industries, northwest region of Florida, 2002 data

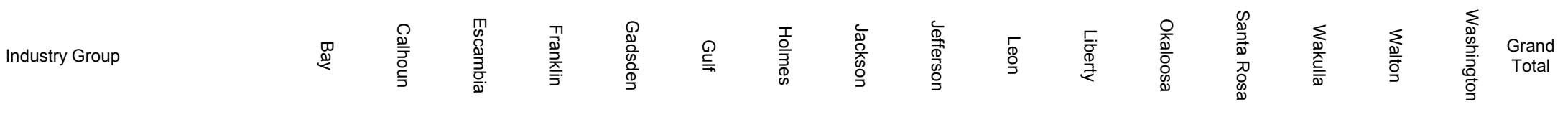

\begin{tabular}{|c|c|c|c|c|c|c|c|c|c|c|c|c|c|c|c|c|c|}
\hline \multirow{2}{*}{$\begin{array}{l}\text { Other Food Product Manufacturing } \\
\text { All other food manufacturing }\end{array}$} & & \multicolumn{16}{|c|}{ (\$Million) } \\
\hline & 0.0 & 0.0 & 0.3 & 0.0 & 0.0 & 0.0 & 0.0 & 0.0 & 0.0 & 0.4 & 0.0 & 0.0 & 0.0 & 0.0 & 0.0 & 0.0 & 0.7 \\
\hline Bread/bakery product, except frozen & 0.0 & 0.0 & 3.8 & 0.0 & 0.0 & 1.7 & 0.0 & 0.2 & 0.0 & 5.5 & 0.0 & 0.8 & 6.4 & 0.0 & 0.0 & 0.0 & 18.3 \\
\hline Coffee \& tea manufacturing & 0.0 & 0.0 & 0.0 & 0.0 & 0.0 & 0.0 & 0.0 & 0.0 & 0.0 & 0.0 & 0.0 & 1.8 & 0.0 & 0.0 & 0.0 & 0.0 & 1.8 \\
\hline Mayonnaise/dressing/sauce mfg & 0.0 & 0.0 & 0.8 & 0.0 & 0.0 & 0.0 & 0.0 & 0.0 & 0.0 & 1.4 & 0.0 & 0.0 & 0.0 & 0.0 & 0.0 & 0.0 & 2.2 \\
\hline Mixes/dough purchased flour & 0.0 & 0.0 & 0.0 & 0.0 & 0.0 & 0.0 & 0.0 & 0.0 & 0.0 & 0.0 & 0.0 & 5.5 & 0.0 & 0.0 & 0.0 & 0.0 & 5.5 \\
\hline Other animal food manufacturing & 0.0 & 0.0 & 1.9 & 0.0 & 0.0 & 0.0 & 0.0 & 2.1 & 0.0 & 0.0 & 0.0 & 0.0 & 0.0 & 0.0 & 0.0 & 0.0 & 4.1 \\
\hline Other snack food manufacturing & 0.0 & 0.0 & 0.0 & 0.0 & 0.0 & 0.0 & 0.0 & 0.0 & 0.0 & 0.0 & 0.0 & 1.1 & 0.0 & 0.0 & 0.0 & 0.0 & 1.1 \\
\hline Soft drink \& ice manufacturing & 3.7 & 0.0 & 5.8 & 0.0 & 0.0 & 0.0 & 0.9 & 0.0 & 0.0 & 7.2 & 0.0 & 0.0 & 0.8 & 1.2 & 0.0 & 0.0 & 19.6 \\
\hline Total & 3.7 & 0.0 & 12.5 & 0.0 & 0.0 & 1.7 & 0.9 & 2.4 & 0.0 & 15.6 & 0.0 & 9.1 & 7.3 & 1.2 & 0.0 & 0.0 & 54.3 \\
\hline Non-chocolate Confectionery Mfg & 0.0 & 0.0 & 0.0 & 0.0 & 0.0 & 0.0 & 0.0 & 0.9 & 0.0 & 0.0 & 0.0 & 0.0 & 0.0 & 0.0 & 0.0 & 0.0 & 0.9 \\
\hline Tobacco Farming \& Manufacturing & 0.0 & 0.0 & 0.0 & 0.0 & 0.0 & 0.0 & 0.0 & 0.0 & 0.6 & 0.5 & 0.0 & 0.0 & 0.0 & 0.0 & 0.0 & 0.0 & 1.1 \\
\hline Wildlife (hunting \& trapping) & 0.0 & 0.0 & 0.0 & 0.0 & 0.0 & 0.0 & 0.0 & 0.0 & 16.7 & 0.0 & 0.0 & 0.0 & 0.0 & 0.0 & 0.0 & 0.0 & 16.7 \\
\hline Total Output Impacts & 388.3 & 74.3 & 719.9 & 45.4 & 361.3 & 22.1 & 93.1 & 254.6 & 106.9 & 152.6 & 60.3 & 114.4 & 297.3 & 38.1 & 205.2 & 68.8 & $3,002.8$ \\
\hline
\end{tabular}

Source: IMPLAN data for Florida counties (2005) 
Table 10. Employment impacts of agriculture and natural resource industries, northwest region of Florida, 2002 data

\begin{tabular}{|c|c|c|c|c|c|c|c|c|c|c|c|c|c|c|c|c|c|}
\hline Industry Group & 罚 & 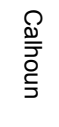 & 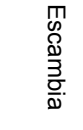 & 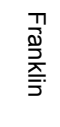 & $\begin{array}{l}\text { Dे } \\
00 \\
00 \\
00 \\
0\end{array}$ & 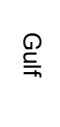 & $\begin{array}{l}\text { T } \\
\frac{\mathrm{O}}{3} \\
\overline{\mathbb{D}} \\
\stackrel{\infty}{\infty}\end{array}$ & 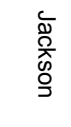 & 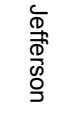 & $\begin{array}{l}\overline{0} \\
\text { อ }\end{array}$ & $\begin{array}{l}\frac{\Gamma}{\overline{0}} \\
\frac{\Phi}{2} \\
\frac{1}{2}\end{array}$ & 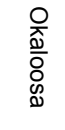 & 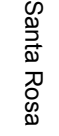 & 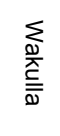 & 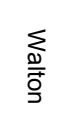 & 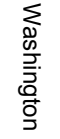 & $\begin{array}{l}\text { Grand } \\
\text { Total }\end{array}$ \\
\hline Agricultural Inputs \& Services & & & & & & & & Jobs & & & & & & & & & \\
\hline Agriculture \& forestry activities & 122 & 9 & 84 & 6 & 350 & 10 & 0 & 59 & 33 & 287 & 6 & 9 & 109 & 3 & 4 & 0 & 1,094 \\
\hline Farm machinery \& equipment mfg & 0 & 0 & 1 & 0 & 4 & 0 & 0 & 0 & 0 & 0 & 0 & 0 & 0 & 0 & 0 & 0 & 6 \\
\hline Fertilizer, mixing only, mfg & 0 & 0 & 2 & 0 & 8 & 0 & 0 & 0 & 0 & 0 & 0 & 0 & 0 & 0 & 0 & 0 & 10 \\
\hline Food product machinery mfg & 0 & 0 & 0 & 0 & 0 & 0 & 0 & 0 & 5 & 0 & 0 & 3 & 0 & 3 & 0 & 0 & 11 \\
\hline Lawn/garden equipment mfg & 0 & 0 & 0 & 0 & 0 & 0 & 0 & 0 & 4 & 0 & 0 & 0 & 0 & 0 & 0 & 0 & 4 \\
\hline New farm housing units/additions & 44 & 3 & 79 & 3 & 7 & 2 & 4 & 6 & 2 & 55 & 3 & 42 & 28 & 5 & 12 & 4 & 300 \\
\hline Phosphatic fertilizer mfg & 0 & 0 & 0 & 0 & 22 & 0 & 0 & 0 & 0 & 0 & 0 & 0 & 0 & 0 & 0 & 0 & 22 \\
\hline Veterinary services & 183 & 4 & 348 & 11 & 58 & 7 & 0 & 17 & 37 & 515 & 0 & 289 & 180 & 19 & 48 & 9 & 1,725 \\
\hline Total & 349 & 17 & 514 & 20 & 450 & 19 & 4 & 82 & 81 & 857 & 9 & 343 & 317 & 31 & 65 & 13 & 3,172 \\
\hline \multicolumn{18}{|l|}{ Environmental Horticulture } \\
\hline Greenhouse /nursery production & 53 & 110 & 84 & 0 & 1,353 & 0 & 98 & 92 & 246 & 78 & 5 & 29 & 137 & 2 & 39 & 9 & 2,335 \\
\hline Landscape services (70\% bldgs) & 779 & 5 & 1,173 & 6 & 159 & 7 & 8 & 54 & 43 & 1,398 & 10 & 799 & 628 & 83 & 259 & 33 & 5,444 \\
\hline Total & 832 & 115 & 1,257 & 6 & 1,512 & 7 & 106 & 146 & 289 & 1,476 & 15 & 827 & 765 & 85 & 298 & 42 & 7,779 \\
\hline \multicolumn{18}{|l|}{ Fishing \& Seafood Products } \\
\hline Fishing & 213 & 0 & 113 & 187 & 0 & 60 & 0 & 0 & 0 & 96 & 0 & 255 & 0 & 0 & 0 & 0 & 925 \\
\hline Seafood product prep/packaging & 82 & 0 & 0 & 207 & 0 & 0 & 0 & 0 & 0 & 0 & 0 & 0 & 0 & 166 & 0 & 0 & 455 \\
\hline Total & 295 & 0 & 113 & 395 & 0 & 60 & 0 & 0 & 0 & 96 & 0 & 255 & 0 & 166 & 0 & 0 & 1,380 \\
\hline \multicolumn{18}{|l|}{ Forestry, Wood \& Paper Product Mfg } \\
\hline Coated \& laminated paper/packaging & 0 & 0 & 263 & 0 & 0 & 0 & 0 & 0 & 0 & 0 & 0 & 0 & 0 & 0 & 0 & 0 & 263 \\
\hline Coated \&uncoated paper bag mfg & 0 & 0 & 715 & 0 & 0 & 0 & 0 & 0 & 0 & 0 & 0 & 0 & 0 & 0 & 0 & 0 & 715 \\
\hline Cut stock/re-sawing lumber/planting & 0 & 19 & 0 & 0 & 0 & 0 & 0 & 0 & 0 & 0 & 0 & 0 & 0 & 0 & 0 & 0 & 19 \\
\hline Engineered wood \& truss mfg & 25 & 0 & 102 & 0 & 624 & 2 & 0 & 0 & 0 & 0 & 0 & 0 & 0 & 0 & 113 & 0 & 866 \\
\hline Envelope manufacturing & 0 & 0 & 8 & 0 & 0 & 0 & 0 & 0 & 0 & 0 & 0 & 0 & 0 & 0 & 0 & 0 & 8 \\
\hline Forest nurseries/products/timber & 70 & 31 & 167 & 0 & 118 & 0 & 0 & 0 & 41 & 23 & 32 & 0 & 114 & 0 & 4 & 23 & 622 \\
\hline Logging & 154 & 207 & 76 & 62 & 6 & 132 & 74 & 115 & 195 & 55 & 200 & 79 & 69 & 0 & 2 & 177 & 1,601 \\
\hline Miscellaneous wood product mfg & 0 & 0 & 0 & 0 & 0 & 0 & 0 & 0 & 0 & 0 & 0 & 8 & 0 & 0 & 0 & 0 & 8 \\
\hline Other millwork, including flooring & 255 & 0 & 5 & 0 & 3 & 0 & 0 & 0 & 0 & 1 & 0 & 4 & 91 & 0 & 0 & 0 & 359 \\
\hline Paper \& paperboard mills & 1,577 & 0 & 2,614 & 0 & 0 & 0 & 0 & 0 & 0 & 0 & 0 & 0 & 0 & 0 & 0 & 0 & 4,190 \\
\hline Paperboard container mfg & 0 & 0 & 61 & 0 & 0 & 0 & 0 & 0 & 0 & 0 & 0 & 0 & 0 & 0 & 0 & 0 & 61 \\
\hline Sawmills & 0 & 109 & 0 & 0 & 0 & 0 & 4 & 0 & 0 & 0 & 6 & 94 & 0 & 0 & 0 & 11 & 225 \\
\hline Veneer \& plywood mfg & 0 & 0 & 0 & 0 & 154 & 0 & 0 & 0 & 0 & 0 & 0 & 0 & 0 & 0 & 0 & 0 & 154 \\
\hline Wood container/pallet mfg & 5 & 0 & 49 & 0 & 0 & 0 & 0 & 4 & 0 & 2 & 0 & 0 & 0 & 0 & 0 & 0 & 60 \\
\hline Wood preservation & 0 & 0 & 7 & 0 & 0 & 0 & 0 & 453 & 0 & 0 & 97 & 0 & 0 & 0 & 0 & 0 & 557 \\
\hline Wood windows/doors mfg & 30 & 0 & 5 & 0 & 0 & 0 & 0 & 3 & 0 & 1 & 0 & 0 & 0 & 0 & 0 & 0 & 39 \\
\hline Total & 2,115 & 366 & 4,071 & 62 & 905 & 135 & 78 & 575 & 237 & 81 & 335 & 185 & 274 & 0 & 120 & 210 & 9,749 \\
\hline
\end{tabular}


Table 10 (continued). Employment Impacts of Agriculture and Natural Resource Industries, Northwest Region of Florida, 2002 Data

\begin{tabular}{|c|c|c|c|c|c|c|c|c|c|c|c|c|c|c|c|c|c|}
\hline Industry Group & $\stackrel{\mathscr{N}}{\aleph}$ & 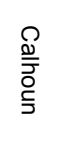 & 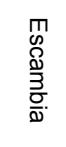 & 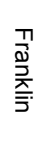 & 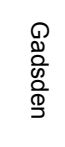 & $\stackrel{\stackrel{\oplus}{\rightleftharpoons}}{\equiv}$ & $\begin{array}{l}\text { T } \\
\text { 을 } \\
\stackrel{\Phi}{\infty}\end{array}$ & 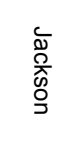 & 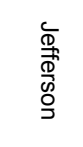 & $\begin{array}{l}\text { \% } \\
\text { O }\end{array}$ & $\begin{array}{l}\bar{\Gamma} \\
\bar{D} \\
\stackrel{D}{\gtrless}\end{array}$ & 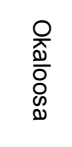 & 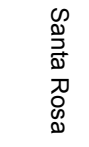 & 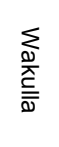 & 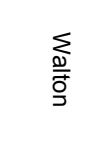 & 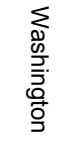 & $\begin{array}{l}\text { Grand } \\
\text { Total }\end{array}$ \\
\hline Fruit \& Vegetable Farming \& Processing & & & & & & & & & Jobs & & & & & & & & \\
\hline Frozen food manufacturing & 11 & 0 & 0 & 0 & 0 & 0 & 0 & 0 & 0 & 0 & 0 & 0 & 0 & 0 & 0 & 0 & 11 \\
\hline Fruit farming & 11 & 2 & 82 & 0 & 93 & 0 & 42 & 75 & 79 & 39 & 0 & 51 & 25 & 1 & 42 & 128 & 669 \\
\hline Vegetable \& melon farming & 2 & 2 & 5 & 0 & 846 & 0 & 36 & 406 & 10 & 6 & 0 & 6 & 9 & 49 & 8 & 47 & 1,434 \\
\hline Total & 25 & 4 & 87 & 0 & 939 & 0 & 77 & 481 & 89 & 46 & 0 & 57 & 34 & 50 & 50 & 175 & 2,114 \\
\hline \multicolumn{18}{|l|}{ Grain \& Oilseed Farming \& Processing } \\
\hline Flour milling & 0 & 0 & 0 & 0 & 0 & 0 & 0 & 0 & 0 & 0 & 0 & 0 & 9 & 0 & 0 & 0 & 9 \\
\hline Grain farming & 2 & 2 & 24 & 0 & 5 & 0 & 5 & 54 & 34 & 6 & 0 & 2 & 3 & 0 & 2 & 19 & 158 \\
\hline Oilseed farming & 0 & 2 & 9 & 0 & 0 & 0 & 2 & 6 & 3 & 0 & 0 & 0 & 5 & 0 & 0 & 6 & 33 \\
\hline Total & 2 & 4 & 32 & 0 & 5 & 0 & 7 & 61 & 37 & 6 & 0 & 2 & 17 & 0 & 2 & 25 & 200 \\
\hline \multicolumn{18}{|c|}{ Livestock \& Dairy Farming \& Animal Products Mfg } \\
\hline Animal production, except cattle \& poultry & 38 & 28 & 114 & 0 & 105 & 0 & 29 & 107 & 7 & 168 & 39 & 198 & 100 & 58 & 228 & 26 & 1,246 \\
\hline Animal, except poultry, slaughtering & 0 & 0 & 39 & 0 & 37 & 0 & 0 & 0 & 0 & 0 & 0 & 0 & 0 & 0 & 0 & 41 & 117 \\
\hline Cattle ranching \& farming & 3 & 31 & 122 & 0 & 39 & 0 & 207 & 405 & 227 & 51 & 3 & 28 & 37 & 2 & 73 & 176 & 1,404 \\
\hline Dry/condensed/evaporated dairy products & 0 & 0 & 0 & 0 & 0 & 0 & 0 & 0 & 0 & 0 & 0 & 1 & 0 & 0 & 0 & 0 & 1 \\
\hline Ice cream \& frozen dessert mfg & 0 & 0 & 2 & 0 & 0 & 0 & 0 & 0 & 0 & 0 & 0 & 0 & 0 & 0 & 0 & 0 & 2 \\
\hline Meat processed from carcasses & 0 & 0 & 2 & 0 & 0 & 0 & 0 & 0 & 0 & 0 & 0 & 0 & 0 & 0 & 0 & 0 & 2 \\
\hline Poultry \& egg production & 0 & 2 & 2 & 0 & 3 & 0 & 208 & 16 & 2 & 0 & 0 & 18 & 0 & 0 & 61 & 5 & 318 \\
\hline Poultry processing & 0 & 0 & 0 & 0 & 0 & 0 & 0 & 0 & 0 & 0 & 0 & 0 & 0 & 0 & 1,001 & 0 & 1,001 \\
\hline Total & 41 & 61 & 280 & 0 & 184 & 0 & 444 & 529 & 237 & 219 & 42 & 246 & 137 & 59 & 1,362 & 248 & 4,091 \\
\hline \multicolumn{18}{|l|}{ Mining } \\
\hline Drilling oil \& gas wells & 0 & 0 & 2 & 0 & 0 & 0 & 0 & 5 & 0 & 0 & 0 & 0 & 7 & 0 & 0 & 0 & 15 \\
\hline Oil \& gas extraction & 66 & 0 & 56 & 0 & 0 & 0 & 0 & 0 & 0 & 0 & 0 & 0 & 2,776 & 0 & 0 & 0 & 2,898 \\
\hline Other nonmetallic mineral mining & 0 & 0 & 0 & 0 & 0 & 0 & 0 & 0 & 0 & 0 & 0 & 0 & 7 & 0 & 0 & 0 & 7 \\
\hline Sand/gravel/clay/refractory mining & 0 & 0 & 115 & 0 & 244 & 0 & 0 & 0 & 0 & 6 & 0 & 6 & 18 & 0 & 5 & 0 & 395 \\
\hline Stone mining \& quarrying & 62 & 0 & 15 & 0 & 0 & 0 & 0 & 98 & 0 & 0 & 0 & 0 & 0 & 0 & 0 & 0 & 175 \\
\hline Support activities for oil \& gas & 0 & 0 & 4 & 0 & 0 & 0 & 0 & 0 & 0 & 5 & 0 & 0 & 16 & 0 & 0 & 0 & 25 \\
\hline Total & 128 & 0 & 192 & 0 & 244 & 0 & 0 & 103 & 0 & 11 & 0 & 6 & 2,824 & 0 & 5 & 0 & 3,514 \\
\hline \multicolumn{18}{|l|}{ Other Crop Farming } \\
\hline All other crop farming & 4 & 16 & 31 & 0 & 10 & 0 & 22 & 180 & 11 & 4 & 0 & 22 & 123 & 0 & 24 & 24 & 470 \\
\hline Cotton farming & 0 & 18 & 62 & 0 & 0 & 0 & 7 & 109 & 3 & 0 & 0 & 12 & 108 & 0 & 5 & 4 & 328 \\
\hline Tree nut farming & 0 & 0 & 3 & 0 & 0 & 0 & 0 & 2 & 2 & 0 & 0 & 0 & 0 & 0 & 0 & 2 & 10 \\
\hline Total & 4 & 33 & 96 & 0 & 10 & 0 & 29 & 292 & 17 & 4 & 0 & 35 & 231 & 0 & 29 & 29 & 808 \\
\hline
\end{tabular}


Table 10 (continued). Employment impacts of agriculture and natural resource industries, northwest region of Florida, 2002 data

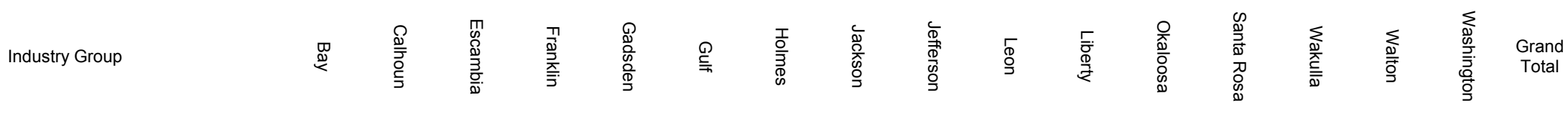

\begin{tabular}{|c|c|c|c|c|c|c|c|c|c|c|c|c|c|c|c|c|c|}
\hline Other Food Product Manufacturing & & & & & & & & & Jobs & & & & & & & & \\
\hline All other food manufacturing & 0 & 0 & 1 & 0 & 0 & 0 & 0 & 0 & 0 & 2 & 0 & 0 & 0 & 0 & 0 & 0 & 3 \\
\hline Bread/bakery, except frozen & 0 & 0 & 53 & 0 & 0 & 18 & 0 & 4 & 0 & 65 & 0 & 10 & 57 & 0 & 0 & 0 & 207 \\
\hline Coffee \& tea manufacturing & 0 & 0 & 0 & 0 & 0 & 0 & 0 & 0 & 0 & 0 & 0 & 4 & 0 & 0 & 0 & 0 & 4 \\
\hline Mayonnaise/dressing/sauce $\mathrm{mfg}$ & 0 & 0 & 3 & 0 & 0 & 0 & 0 & 0 & 0 & 5 & 0 & 0 & 0 & 0 & 0 & 0 & 7 \\
\hline Mixes/dough purchased flour & 0 & 0 & 0 & 0 & 0 & 0 & 0 & 0 & 0 & 0 & 0 & 29 & 0 & 0 & 0 & 0 & 29 \\
\hline Other animal food manufacturing & 0 & 0 & 11 & 0 & 0 & 0 & 0 & 9 & 0 & 0 & 0 & 0 & 0 & 0 & 0 & 0 & 20 \\
\hline Other snack food manufacturing & 0 & 0 & 0 & 0 & 0 & 0 & 0 & 0 & 0 & 0 & 0 & 4 & 0 & 0 & 0 & 0 & 4 \\
\hline Soft drink \& ice manufacturing & 26 & 0 & 41 & 0 & 0 & 0 & 5 & 0 & 0 & 49 & 0 & 0 & 5 & 6 & 0 & 0 & 134 \\
\hline Total & 26 & 0 & 109 & 0 & 0 & 18 & 5 & 13 & 0 & 129 & 0 & 47 & 62 & 6 & 0 & 0 & 416 \\
\hline Non-chocolate Confectionery Mfg & 0 & 0 & 0 & 0 & 0 & 0 & 0 & 8 & 0 & 0 & 0 & 0 & 0 & 0 & 0 & 0 & 8 \\
\hline Tobacco Farming \& Manufacturing & 0 & 0 & 0 & 0 & 0 & 0 & 0 & 0 & 10 & 12 & 0 & 0 & 0 & 0 & 0 & 0 & 22 \\
\hline Wildlife (hunting \& trapping) & 0 & 0 & 0 & 0 & 0 & 0 & 0 & 0 & 233 & 0 & 0 & 0 & 0 & 0 & 0 & 0 & 233 \\
\hline Total Employment Impacts & 3,818 & 600 & 6,752 & 483 & 4,249 & 239 & 751 & 2,291 & 1,229 & 2,936 & 401 & 2,004 & 4,661 & 398 & 1,931 & 743 & 33,486 \\
\hline
\end{tabular}

Source: IMPLAN data for Florida counties (2005) 
Table 11. Value-added Impacts of Agriculture and Natural Resource Industries, Northwest Region of Florida, 2002 Data

\begin{tabular}{|c|c|c|c|c|c|c|c|c|c|c|c|c|c|c|c|c|c|}
\hline Industry Group & $\stackrel{\text { W }}{\stackrel{\mathscr{N}}{\gtrless}}$ & 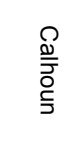 & 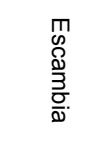 & 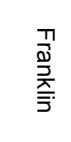 & 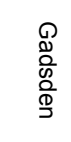 & $\stackrel{\stackrel{\varrho}{\rightleftharpoons}}{\equiv}$ & $\begin{array}{l}\text { I } \\
\frac{0}{3} \\
\mathbb{8} \\
\stackrel{D}{3}\end{array}$ & 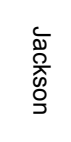 & 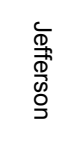 & 힝 & $\begin{array}{l}\bar{\Gamma}: \\
\bar{\Phi} \\
\frac{\mathbb{p}}{2}\end{array}$ & 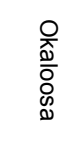 & 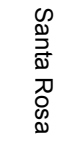 & 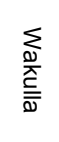 & 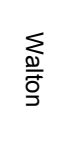 & 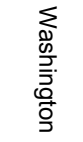 & $\begin{array}{l}\text { Grand } \\
\text { Total }\end{array}$ \\
\hline Agricultural Inputs \& Services & & \multicolumn{16}{|c|}{ (\$Million) } \\
\hline Agriculture \& forestry activities & 4.6 & 0.1 & 4.6 & 0.1 & 6.6 & 0.1 & 0.0 & 0.5 & 0.9 & 15.2 & 0.1 & 0.3 & 4.2 & 0.1 & 0.1 & 0.0 & 37.4 \\
\hline Farm machinery \& equipment mfg & 0.0 & 0.0 & 0.0 & 0.0 & 0.2 & 0.0 & 0.0 & 0.0 & 0.0 & 0.0 & 0.0 & 0.0 & 0.0 & 0.0 & 0.0 & 0.0 & 0.3 \\
\hline Fertilizer, mixing only, mfg & 0.0 & 0.0 & 0.0 & 0.0 & 0.5 & 0.0 & 0.0 & 0.0 & 0.0 & 0.0 & 0.0 & 0.0 & 0.0 & 0.0 & 0.0 & 0.0 & 0.5 \\
\hline Food product machinery mfg & 0.0 & 0.0 & 0.0 & 0.0 & 0.0 & 0.0 & 0.0 & 0.0 & 0.1 & 0.0 & 0.0 & 0.1 & 0.0 & 0.2 & 0.0 & 0.0 & 0.4 \\
\hline Lawn/garden equipment mfg & 0.0 & 0.0 & 0.0 & 0.0 & 0.0 & 0.0 & 0.0 & 0.0 & 0.1 & 0.0 & 0.0 & 0.0 & 0.0 & 0.0 & 0.0 & 0.0 & 0.1 \\
\hline New farm housing units/additions & 1.5 & 0.1 & 2.9 & 0.1 & 0.2 & 0.1 & 0.1 & 0.1 & 0.1 & 2.1 & 0.1 & 1.3 & 0.8 & 0.1 & 0.4 & 0.1 & 10.0 \\
\hline Phosphatic fertilizer mfg & 0.0 & 0.0 & 0.0 & 0.0 & 0.9 & 0.0 & 0.0 & 0.0 & 0.0 & 0.0 & 0.0 & 0.0 & 0.0 & 0.0 & 0.0 & 0.0 & 0.9 \\
\hline Veterinary services & 5.6 & 0.0 & 9.0 & 0.1 & 0.7 & 0.1 & 0.0 & 0.4 & 0.7 & 13.9 & 0.0 & 6.7 & 3.5 & 0.2 & 0.8 & 0.1 & 41.9 \\
\hline Total & 11.6 & 0.2 & 16.5 & 0.2 & 9.2 & 0.3 & 0.1 & 1.0 & 1.9 & 31.1 & 0.2 & 8.4 & 8.6 & 0.6 & 1.3 & 0.2 & 91.6 \\
\hline \multicolumn{18}{|l|}{ Environmental Horticulture } \\
\hline Greenhouse/nursery production & 4.0 & 7.4 & 3.1 & 0.0 & 87.4 & 0.0 & 3.1 & 5.7 & 13.8 & 3.8 & 0.6 & 0.6 & 8.7 & 0.1 & 1.2 & 0.2 & 139.8 \\
\hline Landscape services ( $70 \%$ bldgs) & 18.6 & 0.1 & 21.5 & 0.1 & 4.7 & 0.2 & 0.3 & 0.8 & 1.0 & 25.1 & 0.2 & 18.2 & 12.7 & 2.0 & 7.7 & 0.4 & 113.6 \\
\hline Total & 22.6 & 7.5 & 24.6 & 0.1 & 92.1 & 0.2 & 3.4 & 6.6 & 14.8 & 28.8 & 0.8 & 18.8 & 21.4 & 2.1 & 8.9 & 0.7 & 253.5 \\
\hline \multicolumn{18}{|l|}{ Fishing \& Seafood Products } \\
\hline Fishing & 4.6 & 0.0 & 4.0 & 4.3 & 0.0 & 0.7 & 0.0 & 0.0 & 0.0 & 1.6 & 0.0 & 3.0 & 0.0 & 0.0 & 0.0 & 0.0 & 18.2 \\
\hline Seafood product prep/packaging & 3.9 & 0.0 & 0.0 & 8.1 & 0.0 & 0.0 & 0.0 & 0.0 & 0.0 & 0.0 & 0.0 & 0.0 & 0.0 & 3.0 & 0.0 & 0.0 & 15.1 \\
\hline Total & 8.5 & 0.0 & 4.0 & 12.4 & 0.0 & 0.7 & 0.0 & 0.0 & 0.0 & 1.6 & 0.0 & 3.0 & 0.0 & 3.0 & 0.0 & 0.0 & 33.3 \\
\hline \multicolumn{18}{|l|}{ Forestry, Wood \& Paper Product Mfg } \\
\hline Coated \& laminated paper/packaging & 0.0 & 0.0 & 17.1 & 0.0 & 0.0 & 0.0 & 0.0 & 0.0 & 0.0 & 0.0 & 0.0 & 0.0 & 0.0 & 0.0 & 0.0 & 0.0 & 17.1 \\
\hline Coated \&uncoated paper bag mfg & 0.0 & 0.0 & 38.8 & 0.0 & 0.0 & 0.0 & 0.0 & 0.0 & 0.0 & 0.0 & 0.0 & 0.0 & 0.0 & 0.0 & 0.0 & 0.0 & 38.8 \\
\hline Cut stock/re-sawing lumber/planting & 0.0 & 0.8 & 0.0 & 0.0 & 0.0 & 0.0 & 0.0 & 0.0 & 0.0 & 0.0 & 0.0 & 0.0 & 0.0 & 0.0 & 0.0 & 0.0 & 0.8 \\
\hline Engineered wood \& truss mfg & 1.2 & 0.0 & 4.7 & 0.0 & 34.1 & 0.1 & 0.0 & 0.0 & 0.0 & 0.0 & 0.0 & 0.0 & 0.0 & 0.0 & 4.9 & 0.0 & 45.0 \\
\hline Envelope manufacturing & 0.0 & 0.0 & 0.5 & 0.0 & 0.0 & 0.0 & 0.0 & 0.0 & 0.0 & 0.0 & 0.0 & 0.0 & 0.0 & 0.0 & 0.0 & 0.0 & 0.5 \\
\hline Forest nurseries/products/timber & 3.8 & 1.3 & 12.8 & 0.0 & 3.9 & 0.0 & 0.0 & 0.0 & 2.0 & 1.1 & 1.5 & 0.0 & 6.0 & 0.0 & 0.2 & 1.0 & 33.7 \\
\hline Logging & 10.4 & 12.8 & 11.9 & 3.1 & 0.3 & 6.4 & 6.4 & 5.3 & 9.8 & 2.8 & 12.4 & 6.2 & 4.4 & 0.0 & 0.2 & 9.0 & 101.3 \\
\hline Miscellaneous wood product mfg & 0.0 & 0.0 & 0.0 & 0.0 & 0.0 & 0.0 & 0.0 & 0.0 & 0.0 & 0.0 & 0.0 & 0.4 & 0.0 & 0.0 & 0.0 & 0.0 & 0.4 \\
\hline Other millwork, including flooring & 10.5 & 0.0 & 0.2 & 0.0 & 0.1 & 0.0 & 0.0 & 0.0 & 0.0 & 0.0 & 0.0 & 0.1 & 3.0 & 0.0 & 0.0 & 0.0 & 13.9 \\
\hline Paper \& paperboard mills & 105.9 & 0.0 & 178.2 & 0.0 & 0.0 & 0.0 & 0.0 & 0.0 & 0.0 & 0.0 & 0.0 & 0.0 & 0.0 & 0.0 & 0.0 & 0.0 & 284.1 \\
\hline Paperboard container mfg & 0.0 & 0.0 & 3.2 & 0.0 & 0.0 & 0.0 & 0.0 & 0.0 & 0.0 & 0.0 & 0.0 & 0.0 & 0.0 & 0.0 & 0.0 & 0.0 & 3.2 \\
\hline Sawmills & 0.0 & 5.2 & 0.0 & 0.0 & 0.0 & 0.0 & 0.2 & 0.0 & 0.0 & 0.0 & 0.2 & 4.6 & 0.0 & 0.0 & 0.0 & 0.5 & 10.8 \\
\hline Veneer \& plywood mfg & 0.0 & 0.0 & 0.0 & 0.0 & 6.8 & 0.0 & 0.0 & 0.0 & 0.0 & 0.0 & 0.0 & 0.0 & 0.0 & 0.0 & 0.0 & 0.0 & 6.8 \\
\hline Wood container/pallet mfg & 0.2 & 0.0 & 1.8 & 0.0 & 0.0 & 0.0 & 0.0 & 0.1 & 0.0 & 0.0 & 0.0 & 0.0 & 0.0 & 0.0 & 0.0 & 0.0 & 2.1 \\
\hline Wood preservation & 0.0 & 0.0 & 0.3 & 0.0 & 0.0 & 0.0 & 0.0 & 19.8 & 0.0 & 0.0 & 5.4 & 0.0 & 0.0 & 0.0 & 0.0 & 0.0 & 25.5 \\
\hline Wood windows/doors $\mathrm{mfg}$ & 1.6 & 0.0 & 0.3 & 0.0 & 0.0 & 0.0 & 0.0 & 0.1 & 0.0 & 0.0 & 0.0 & 0.0 & 0.0 & 0.0 & 0.0 & 0.0 & 2.0 \\
\hline Total & 133.7 & 20.1 & 269.8 & 3.1 & 45.1 & 6.5 & 6.5 & 25.2 & 11.8 & 4.0 & 19.6 & 11.3 & 13.5 & 0.0 & 5.4 & 10.5 & 586.0 \\
\hline
\end{tabular}


Table 11(continued). Value-added impacts of agriculture and natural resource industries, northwest region of Florida, 2002 data

\begin{tabular}{|c|c|c|c|c|c|c|c|c|c|c|c|c|c|c|c|c|c|}
\hline Industry Group & 罚 & 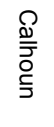 & 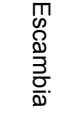 & 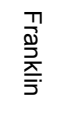 & $\begin{array}{l}0 \\
\stackrel{D}{0} \\
\frac{0}{00} \\
\frac{0}{0} \\
\stackrel{D}{I}\end{array}$ & 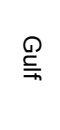 & $\begin{array}{l}\text { T } \\
\text { 을 } \\
\text { ర్ల } \\
\infty\end{array}$ & 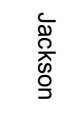 & 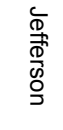 & $\begin{array}{l}\frac{1}{8} \\
\end{array}$ & 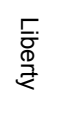 & 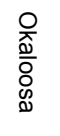 & $\begin{array}{l}\infty \\
\infty \\
\beth_{D} \\
D \\
0 \\
0 \\
\infty\end{array}$ & 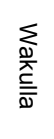 & 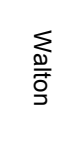 & 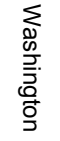 & $\begin{array}{l}\text { Grand } \\
\text { Total }\end{array}$ \\
\hline Fruit \& Vegetable Farming \& Processing & & \multicolumn{16}{|c|}{ (\$Million) } \\
\hline Frozen food manufacturing & 0.6 & 0.0 & 0.0 & 0.0 & 0.0 & 0.0 & 0.0 & 0.0 & 0.0 & 0.0 & 0.0 & 0.0 & 0.0 & 0.0 & 0.0 & 0.0 & 0.6 \\
\hline Fruit farming & 0.5 & 0.1 & 1.4 & 0.0 & 3.4 & 0.0 & 0.5 & 2.1 & 2.2 & 1.2 & 0.0 & 0.5 & 0.7 & 0.0 & 0.6 & 1.9 & 15.0 \\
\hline Vegetable \& melon farming & 0.2 & 0.2 & 0.3 & 0.0 & 47.4 & 0.0 & 1.7 & 28.7 & 0.7 & 0.3 & 0.0 & 0.2 & 0.9 & 4.0 & 0.3 & 1.9 & 86.6 \\
\hline Total & 1.3 & 0.3 & 1.7 & 0.0 & 50.8 & 0.0 & 2.2 & 30.8 & 2.9 & 1.4 & 0.0 & 0.7 & 1.5 & 4.0 & 0.9 & 3.7 & 102.2 \\
\hline \multicolumn{18}{|l|}{ Grain \& Oilseed Farming \& Processing } \\
\hline Flour milling & 0.0 & 0.0 & 0.0 & 0.0 & 0.0 & 0.0 & 0.0 & 0.0 & 0.0 & 0.0 & 0.0 & 0.0 & 0.5 & 0.0 & 0.0 & 0.0 & 0.5 \\
\hline Grain farming & 0.1 & 0.1 & 0.6 & 0.0 & 0.1 & 0.0 & 0.1 & 2.2 & 0.9 & 0.1 & 0.0 & 0.0 & 0.1 & 0.0 & 0.1 & 0.3 & 4.8 \\
\hline Oilseed farming & 0.0 & 0.1 & 0.4 & 0.0 & 0.0 & 0.0 & 0.1 & 0.4 & 0.1 & 0.0 & 0.0 & 0.0 & 0.2 & 0.0 & 0.0 & 0.2 & 1.5 \\
\hline Total & 0.1 & 0.2 & 1.0 & 0.0 & 0.1 & 0.0 & 0.2 & 2.6 & 1.1 & 0.1 & 0.0 & 0.0 & 0.8 & 0.0 & 0.1 & 0.5 & 6.7 \\
\hline \multicolumn{18}{|c|}{ Livestock \& Dairy Farming \& Animal Products Mfg } \\
\hline Animal production, except cattle \& poultry & 0.2 & 0.2 & 0.3 & 0.0 & 1.2 & 0.0 & 0.1 & 0.9 & 0.0 & 1.8 & 0.2 & 1.0 & 0.7 & 0.2 & 1.6 & 0.0 & 8.5 \\
\hline Animal, except poultry, slaughtering & 0.0 & 0.0 & 1.0 & 0.0 & 1.2 & 0.0 & 0.0 & 0.0 & 0.0 & 0.0 & 0.0 & 0.0 & 0.0 & 0.0 & 0.0 & 1.0 & 3.2 \\
\hline Cattle ranching \& farming & 0.1 & 0.5 & 1.9 & 0.0 & 0.5 & 0.0 & 1.6 & 6.7 & 3.0 & 1.1 & 0.0 & 0.5 & 0.7 & 0.0 & 1.2 & 1.6 & 19.1 \\
\hline Dry/condensed/evaporated dairy products & 0.0 & 0.0 & 0.0 & 0.0 & 0.0 & 0.0 & 0.0 & 0.0 & 0.0 & 0.0 & 0.0 & 0.1 & 0.0 & 0.0 & 0.0 & 0.0 & 0.1 \\
\hline Ice cream \& frozen dessert mfg & 0.0 & 0.0 & 0.1 & 0.0 & 0.0 & 0.0 & 0.0 & 0.0 & 0.0 & 0.0 & 0.0 & 0.0 & 0.0 & 0.0 & 0.0 & 0.0 & 0.1 \\
\hline Meat processed from carcasses & 0.0 & 0.0 & 0.0 & 0.0 & 0.0 & 0.0 & 0.0 & 0.0 & 0.0 & 0.0 & 0.0 & 0.0 & 0.0 & 0.0 & 0.0 & 0.0 & 0.0 \\
\hline Poultry \& egg production & 0.0 & 0.2 & 0.1 & 0.0 & 0.3 & 0.0 & 16.5 & 1.7 & 0.2 & 0.0 & 0.0 & 1.1 & 0.0 & 0.0 & 5.8 & 0.3 & 26.3 \\
\hline Poultry processing & 0.0 & 0.0 & 0.0 & 0.0 & 0.0 & 0.0 & 0.0 & 0.0 & 0.0 & 0.0 & 0.0 & 0.0 & 0.0 & 0.0 & 42.4 & 0.0 & 42.4 \\
\hline Total & 0.2 & 0.9 & 3.4 & 0.0 & 3.1 & 0.0 & 18.2 & 9.3 & 3.3 & 2.9 & 0.3 & 2.6 & 1.3 & 0.2 & 50.9 & 2.9 & 99.6 \\
\hline \multicolumn{18}{|l|}{ Mining } \\
\hline Drilling oil \& gas wells & 0.0 & 0.0 & 0.1 & 0.0 & 0.0 & 0.0 & 0.0 & 0.1 & 0.0 & 0.0 & 0.0 & 0.0 & 0.2 & 0.0 & 0.0 & 0.0 & 0.3 \\
\hline Oil \& gas extraction & 0.4 & 0.0 & 2.2 & 0.0 & 0.0 & 0.0 & 0.0 & 0.0 & 0.0 & 0.0 & 0.0 & 0.0 & 97.3 & 0.0 & 0.0 & 0.0 & 99.8 \\
\hline Other nonmetallic mineral mining & 0.0 & 0.0 & 0.0 & 0.0 & 0.0 & 0.0 & 0.0 & 0.0 & 0.0 & 0.0 & 0.0 & 0.0 & 0.3 & 0.0 & 0.0 & 0.0 & 0.3 \\
\hline Sand/gravel/clay/refractory mining & 0.0 & 0.0 & 7.6 & 0.0 & 18.0 & 0.0 & 0.0 & 0.0 & 0.0 & 0.2 & 0.0 & 0.4 & 1.1 & 0.0 & 0.5 & 0.0 & 27.8 \\
\hline Stone mining \& quarrying & 3.8 & 0.0 & 0.7 & 0.0 & 0.0 & 0.0 & 0.0 & 6.0 & 0.0 & 0.0 & 0.0 & 0.0 & 0.0 & 0.0 & 0.0 & 0.0 & 10.5 \\
\hline Support activities for oil \& gas & 0.0 & 0.0 & 0.1 & 0.0 & 0.0 & 0.0 & 0.0 & 0.0 & 0.0 & 0.1 & 0.0 & 0.0 & 0.4 & 0.0 & 0.0 & 0.0 & 0.6 \\
\hline Total & 4.2 & 0.0 & 10.7 & 0.0 & 18.0 & 0.0 & 0.0 & 6.0 & 0.0 & 0.4 & 0.0 & 0.4 & 99.3 & 0.0 & 0.5 & 0.0 & 139.4 \\
\hline \multicolumn{18}{|l|}{ Other Crop Farming } \\
\hline All other crop farming & 0.2 & 1.1 & 1.3 & 0.0 & 0.5 & 0.0 & 0.8 & 11.6 & 0.6 & 0.2 & 0.0 & 0.6 & 7.5 & 0.0 & 0.8 & 0.7 & 25.8 \\
\hline Cotton farming & 0.0 & 1.1 & 2.9 & 0.0 & 0.0 & 0.0 & 0.2 & 5.6 & 0.2 & 0.0 & 0.0 & 0.4 & 6.1 & 0.0 & 0.2 & 0.1 & 16.9 \\
\hline Tree nut farming & 0.0 & 0.0 & 0.1 & 0.0 & 0.0 & 0.0 & 0.0 & 0.1 & 0.1 & 0.0 & 0.0 & 0.0 & 0.0 & 0.0 & 0.0 & 0.0 & 0.5 \\
\hline Total & 0.2 & 2.2 & 4.4 & 0.0 & 0.5 & 0.0 & 1.0 & 17.3 & 1.0 & 0.2 & 0.0 & 1.0 & 13.6 & 0.0 & 0.9 & 0.9 & 43.2 \\
\hline
\end{tabular}


Table 11(continued). Value-added impacts of agriculture and natural resource industries, northwest region of Florida, 2002 data

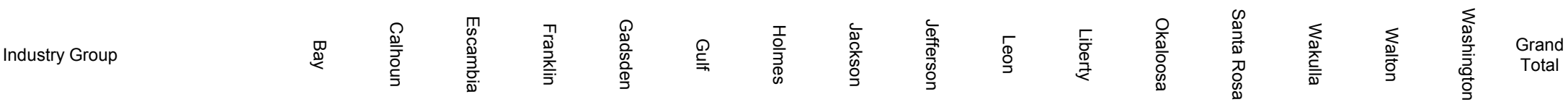

\begin{tabular}{|c|c|c|c|c|c|c|c|c|c|c|c|c|c|c|c|c|c|}
\hline \multirow{2}{*}{$\begin{array}{l}\text { Other Food Product Manufacturing } \\
\text { All other food manufacturing }\end{array}$} & & \multicolumn{16}{|c|}{ (\$Million) } \\
\hline & 0.0 & 0.0 & 0.0 & 0.0 & 0.0 & 0.0 & 0.0 & 0.0 & 0.0 & 0.1 & 0.0 & 0.0 & 0.0 & 0.0 & 0.0 & 0.0 & 0.1 \\
\hline Bread/bakery, except frozen & 0.0 & 0.0 & 1.6 & 0.0 & 0.0 & 0.9 & 0.0 & 0.1 & 0.0 & 2.6 & 0.0 & 0.3 & 3.5 & 0.0 & 0.0 & 0.0 & 9.0 \\
\hline Coffee \& tea manufacturing & 0.0 & 0.0 & 0.0 & 0.0 & 0.0 & 0.0 & 0.0 & 0.0 & 0.0 & 0.0 & 0.0 & 0.2 & 0.0 & 0.0 & 0.0 & 0.0 & 0.2 \\
\hline Mayonnaise/dressing/sauce mfg & 0.0 & 0.0 & 0.1 & 0.0 & 0.0 & 0.0 & 0.0 & 0.0 & 0.0 & 0.2 & 0.0 & 0.0 & 0.0 & 0.0 & 0.0 & 0.0 & 0.3 \\
\hline Mixes/dough purchased flour & 0.0 & 0.0 & 0.0 & 0.0 & 0.0 & 0.0 & 0.0 & 0.0 & 0.0 & 0.0 & 0.0 & 2.3 & 0.0 & 0.0 & 0.0 & 0.0 & 2.3 \\
\hline Other animal food $\mathrm{mfg}$ & 0.0 & 0.0 & 0.5 & 0.0 & 0.0 & 0.0 & 0.0 & 0.4 & 0.0 & 0.0 & 0.0 & 0.0 & 0.0 & 0.0 & 0.0 & 0.0 & 0.9 \\
\hline Other snack food manufacturing & 0.0 & 0.0 & 0.0 & 0.0 & 0.0 & 0.0 & 0.0 & 0.0 & 0.0 & 0.0 & 0.0 & 0.3 & 0.0 & 0.0 & 0.0 & 0.0 & 0.3 \\
\hline Soft drink \& ice manufacturing & 1.5 & 0.0 & 2.6 & 0.0 & 0.0 & 0.0 & 0.2 & 0.0 & 0.0 & 2.6 & 0.0 & 0.0 & 0.2 & 0.3 & 0.0 & 0.0 & 7.4 \\
\hline Total & 1.5 & 0.0 & 4.9 & 0.0 & 0.0 & 0.9 & 0.2 & 0.5 & 0.0 & 6.2 & 0.0 & 3.2 & 3.7 & 0.3 & 0.0 & 0.0 & 21.3 \\
\hline Non-chocolate Confectionery Mfg & 0.0 & 0.0 & 0.0 & 0.0 & 0.0 & 0.0 & 0.0 & 0.3 & 0.0 & 0.0 & 0.0 & 0.0 & 0.0 & 0.0 & 0.0 & 0.0 & 0.3 \\
\hline Tobacco Farming \& Manufacturing & 0.0 & 0.0 & 0.0 & 0.0 & 0.0 & 0.0 & 0.0 & 0.0 & 0.4 & 0.3 & 0.0 & 0.0 & 0.0 & 0.0 & 0.0 & 0.0 & 0.7 \\
\hline Wildlife (hunting \& trapping) & 0.0 & 0.0 & 0.0 & 0.0 & 0.0 & 0.0 & 0.0 & 0.0 & 7.9 & 0.0 & 0.0 & 0.0 & 0.0 & 0.0 & 0.0 & 0.0 & 7.9 \\
\hline Total Employment Impacts & 184.0 & 31.5 & 340.8 & 15.9 & 219.1 & 8.6 & 31.8 & 99.5 & 44.9 & 77.0 & 20.8 & 49.5 & 163.7 & 10.2 & 68.8 & 19.4 & $1,385.6$ \\
\hline
\end{tabular}

Source: IMPLAN data for Florida counties (2005) 
Table 12. Labor Income Impacts of Agriculture and Natural Resource Industries, Northwest Region of Florida, 2002 Data

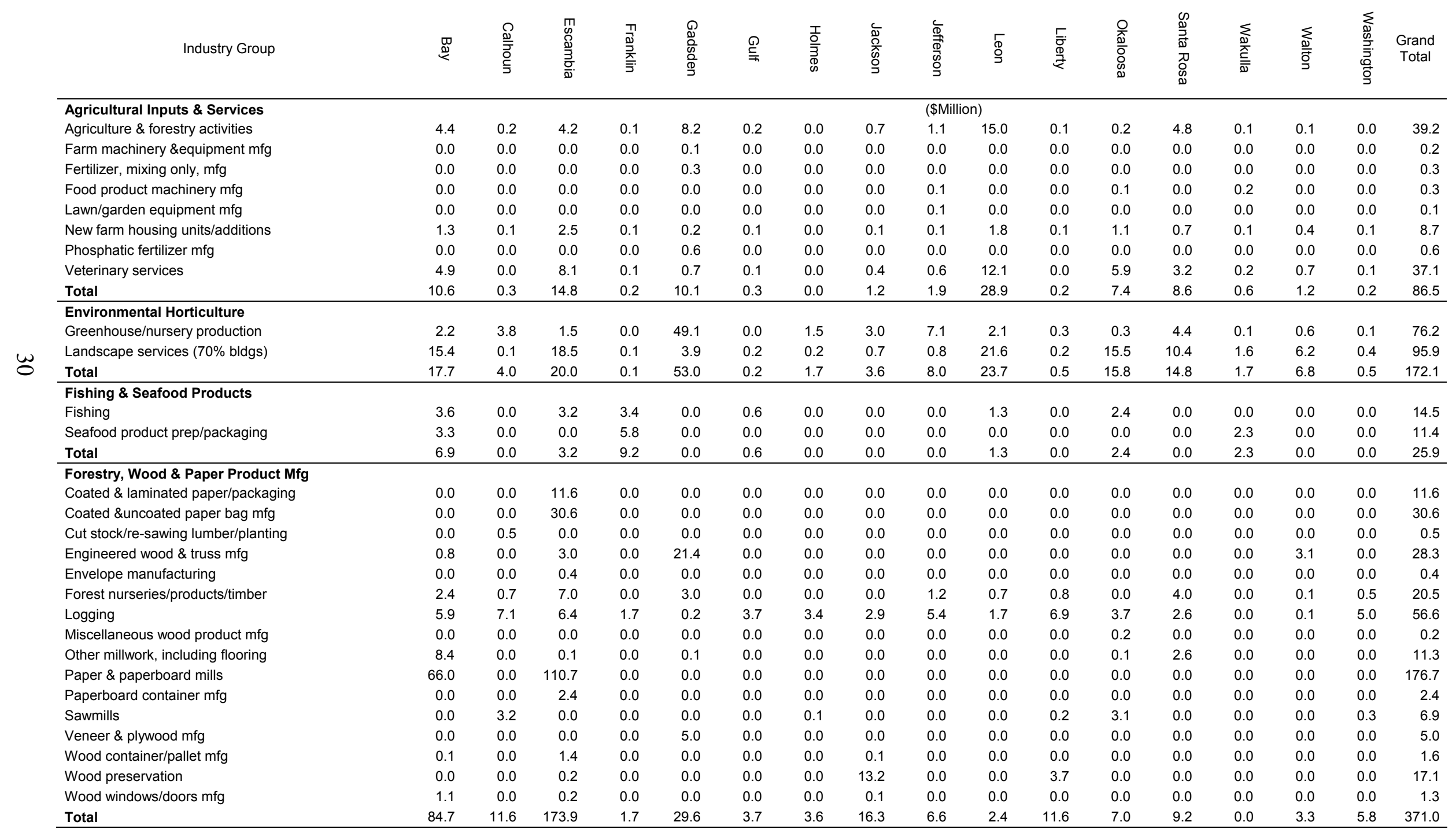


Table 12 (continued). Labor Income Impacts of Agriculture and Natural Resource Industries, Northwest Region of Florida, 2002 Data

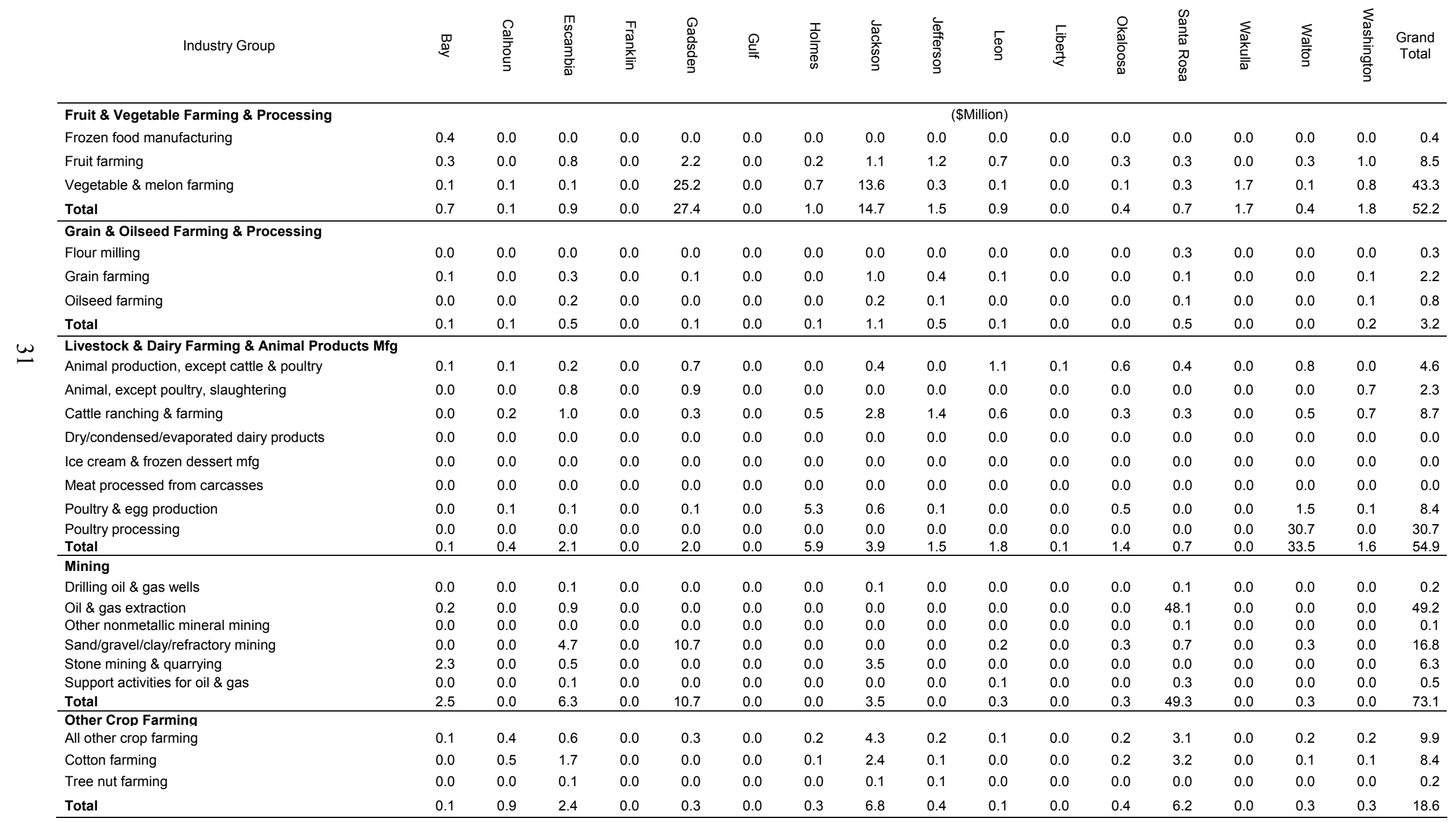


Table 12 (continued). Labor income impacts of agriculture and natural resource industries, northwest region of Florida, 2002 data

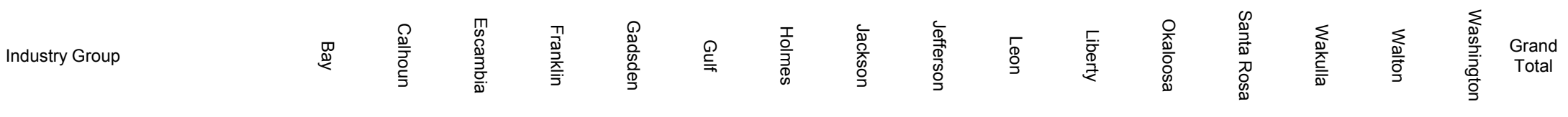

\begin{tabular}{|c|c|c|c|c|c|c|c|c|c|c|c|c|c|c|c|c|c|}
\hline \multirow{2}{*}{$\begin{array}{l}\text { Other Food Product Manufacturing } \\
\text { All other food manufacturing }\end{array}$} & & & \multicolumn{15}{|c|}{ (\$Million) } \\
\hline & 0.0 & 0.0 & 0.0 & 0.0 & 0.0 & 0.0 & 0.0 & 0.0 & 0.0 & 0.0 & 0.0 & 0.0 & 0.0 & 0.0 & 0.0 & 0.0 & 0.1 \\
\hline Bread/bakery, except frozen & 0.0 & 0.0 & 0.9 & 0.0 & 0.0 & 0.5 & 0.0 & 0.1 & 0.0 & 1.5 & 0.0 & 0.2 & 2.0 & 0.0 & 0.0 & 0.0 & 5.2 \\
\hline Coffee \& tea manufacturing & 0.0 & 0.0 & 0.0 & 0.0 & 0.0 & 0.0 & 0.0 & 0.0 & 0.0 & 0.0 & 0.0 & 0.1 & 0.0 & 0.0 & 0.0 & 0.0 & 0.1 \\
\hline Mayonnaise/dressing/sauce mfg & 0.0 & 0.0 & 0.0 & 0.0 & 0.0 & 0.0 & 0.0 & 0.0 & 0.0 & 0.1 & 0.0 & 0.0 & 0.0 & 0.0 & 0.0 & 0.0 & 0.1 \\
\hline Mixes/dough purchased flour & 0.0 & 0.0 & 0.0 & 0.0 & 0.0 & 0.0 & 0.0 & 0.0 & 0.0 & 0.0 & 0.0 & 1.2 & 0.0 & 0.0 & 0.0 & 0.0 & 1.2 \\
\hline Other animal food manufacturing & 0.0 & 0.0 & 0.3 & 0.0 & 0.0 & 0.0 & 0.0 & 0.2 & 0.0 & 0.0 & 0.0 & 0.0 & 0.0 & 0.0 & 0.0 & 0.0 & 0.6 \\
\hline Other snack food manufacturing & 0.0 & 0.0 & 0.0 & 0.0 & 0.0 & 0.0 & 0.0 & 0.0 & 0.0 & 0.0 & 0.0 & 0.1 & 0.0 & 0.0 & 0.0 & 0.0 & 0.1 \\
\hline Soft drink \& ice manufacturing & 0.9 & 0.0 & 1.7 & 0.0 & 0.0 & 0.0 & 0.1 & 0.0 & 0.0 & 1.6 & 0.0 & 0.0 & 0.1 & 0.2 & 0.0 & 0.0 & 4.7 \\
\hline Total & 0.9 & 0.0 & 3.0 & 0.0 & 0.0 & 0.5 & 0.1 & 0.3 & 0.0 & 3.4 & 0.0 & 1.6 & 2.1 & 0.2 & 0.0 & 0.0 & 12.2 \\
\hline Non-chocolate Confectionery Mfg & 0.0 & 0.0 & 0.0 & 0.0 & 0.0 & 0.0 & 0.0 & 0.1 & 0.0 & 0.0 & 0.0 & 0.0 & 0.0 & 0.0 & 0.0 & 0.0 & 0.1 \\
\hline Tobacco Farming \& Manufacturing & 0.0 & 0.0 & 0.0 & 0.0 & 0.0 & 0.0 & 0.0 & 0.0 & 0.2 & 0.2 & 0.0 & 0.0 & 0.0 & 0.0 & 0.0 & 0.0 & 0.3 \\
\hline Wildlife (hunting \& trapping) & 0.0 & 0.0 & 0.0 & 0.0 & 0.0 & 0.0 & 0.0 & 0.0 & 3.7 & 0.0 & 0.0 & 0.0 & 0.0 & 0.0 & 0.0 & 0.0 & 3.7 \\
\hline Total Employment Impacts & 124.3 & 17.4 & 227.2 & 11.3 & 133.1 & 5.3 & 12.6 & 51.6 & 24.2 & 63.0 & 12.3 & 36.8 & 92.1 & 6.5 & 45.8 & 10.5 & 873.9 \\
\hline
\end{tabular}

Source: IMPLAN data for Florida counties (2005) 
Table 13. Indirect business tax impacts of agriculture and natural resource industries, northwest region of Florida, 2002 data

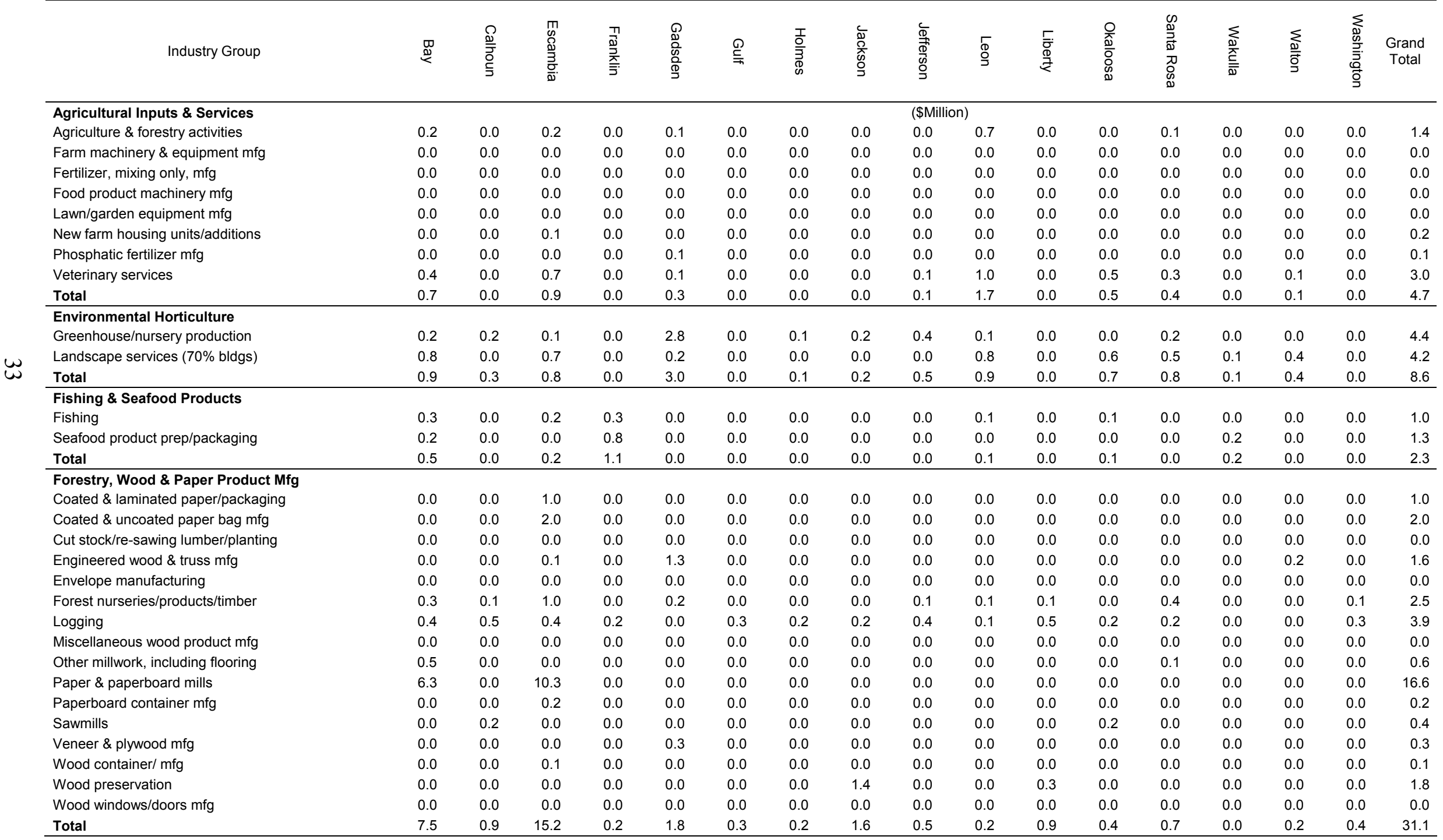


Table 13 (continued). Indirect business tax impacts of agriculture and natural resource industries, northwest region of Florida, 2002 data

\begin{tabular}{|c|c|c|c|c|c|c|c|c|c|c|c|c|c|c|c|c|c|}
\hline Industry Group & 罚 & $\begin{array}{l}\stackrel{\Omega}{\mathscr{N}} \\
\overline{\bar{O}} \\
\subseteq\end{array}$ & 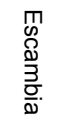 & 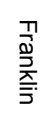 & $\begin{array}{l}\stackrel{Q}{0} \\
\stackrel{0}{0} \\
\stackrel{0}{D} \\
\stackrel{D}{I}\end{array}$ & $\stackrel{\oplus}{\risingdotseq}$ & 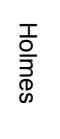 & 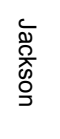 & 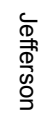 & 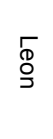 & 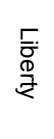 & 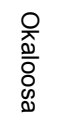 & $\begin{array}{l}\infty \\
\infty \\
\bar{D} \\
\mathbb{D} \\
D \\
0 \\
\infty \\
\Phi\end{array}$ & 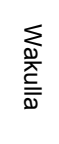 & 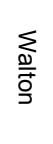 & 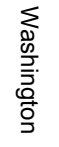 & $\begin{array}{l}\text { Grand } \\
\text { Total }\end{array}$ \\
\hline Fruit \& Vegetable Farming \& Processing & & \multicolumn{16}{|c|}{ (\$Million) } \\
\hline Frozen food manufacturing & 0.0 & 0.0 & 0.0 & 0.0 & 0.0 & 0.0 & 0.0 & 0.0 & 0.0 & 0.0 & 0.0 & 0.0 & 0.0 & 0.0 & 0.0 & 0.0 & 0.0 \\
\hline Fruit farming & 0.0 & 0.0 & 0.1 & 0.0 & 0.2 & 0.0 & 0.0 & 0.1 & 0.1 & 0.1 & 0.0 & 0.0 & 0.0 & 0.0 & 0.0 & 0.1 & 0.9 \\
\hline Vegetable \& melon farming & 0.0 & 0.0 & 0.0 & 0.0 & 1.8 & 0.0 & 0.1 & 1.2 & 0.0 & 0.0 & 0.0 & 0.0 & 0.0 & 0.1 & 0.0 & 0.1 & 3.3 \\
\hline Total & 0.1 & 0.0 & 0.1 & 0.0 & 2.0 & 0.0 & 0.1 & 1.3 & 0.2 & 0.1 & 0.0 & 0.0 & 0.1 & 0.1 & 0.1 & 0.2 & 4.3 \\
\hline \multicolumn{18}{|l|}{ Grain \& Oilseed Farming \& Processing } \\
\hline Flour milling & 0.0 & 0.0 & 0.0 & 0.0 & 0.0 & 0.0 & 0.0 & 0.0 & 0.0 & 0.0 & 0.0 & 0.0 & 0.0 & 0.0 & 0.0 & 0.0 & 0.0 \\
\hline Grain farming & 0.0 & 0.0 & 0.0 & 0.0 & 0.0 & 0.0 & 0.0 & 0.1 & 0.1 & 0.0 & 0.0 & 0.0 & 0.0 & 0.0 & 0.0 & 0.0 & 0.3 \\
\hline Oilseed farming & 0.0 & 0.0 & 0.0 & 0.0 & 0.0 & 0.0 & 0.0 & 0.0 & 0.0 & 0.0 & 0.0 & 0.0 & 0.0 & 0.0 & 0.0 & 0.0 & 0.1 \\
\hline Total & 0.0 & 0.0 & 0.1 & 0.0 & 0.0 & 0.0 & 0.0 & 0.2 & 0.1 & 0.0 & 0.0 & 0.0 & 0.1 & 0.0 & 0.0 & 0.0 & 0.4 \\
\hline \multicolumn{18}{|l|}{ Livestock \& Dairy Farming \& Animal Products Mfg } \\
\hline Animal production, except cattle \& poultry & 0.0 & 0.0 & 0.0 & 0.0 & 0.1 & 0.0 & 0.0 & 0.1 & 0.0 & 0.2 & 0.0 & 0.1 & 0.1 & 0.1 & 0.1 & 0.0 & 1.1 \\
\hline Animal, except poultry, slaughtering & 0.0 & 0.0 & 0.1 & 0.0 & 0.1 & 0.0 & 0.0 & 0.0 & 0.0 & 0.0 & 0.0 & 0.0 & 0.0 & 0.0 & 0.0 & 0.1 & 0.3 \\
\hline Cattle ranching \& farming & 0.0 & 0.1 & 0.2 & 0.0 & 0.1 & 0.0 & 0.2 & 0.8 & 0.5 & 0.2 & 0.0 & 0.0 & 0.1 & 0.0 & 0.1 & 0.2 & 2.5 \\
\hline Dry/condensed/evaporated dairy products & 0.0 & 0.0 & 0.0 & 0.0 & 0.0 & 0.0 & 0.0 & 0.0 & 0.0 & 0.0 & 0.0 & 0.0 & 0.0 & 0.0 & 0.0 & 0.0 & 0.0 \\
\hline Ice cream \& frozen dessert mfg & 0.0 & 0.0 & 0.0 & 0.0 & 0.0 & 0.0 & 0.0 & 0.0 & 0.0 & 0.0 & 0.0 & 0.0 & 0.0 & 0.0 & 0.0 & 0.0 & 0.0 \\
\hline Meat processed from carcasses & 0.0 & 0.0 & 0.0 & 0.0 & 0.0 & 0.0 & 0.0 & 0.0 & 0.0 & 0.0 & 0.0 & 0.0 & 0.0 & 0.0 & 0.0 & 0.0 & 0.0 \\
\hline Poultry \& egg production & 0.0 & 0.0 & 0.0 & 0.0 & 0.0 & 0.0 & 0.5 & 0.1 & 0.0 & 0.0 & 0.0 & 0.0 & 0.0 & 0.0 & 0.1 & 0.0 & 0.8 \\
\hline Poultry processing & 0.0 & 0.0 & 0.0 & 0.0 & 0.0 & 0.0 & 0.0 & 0.0 & 0.0 & 0.0 & 0.0 & 0.0 & 0.0 & 0.0 & 2.6 & 0.0 & 2.6 \\
\hline Total & 0.1 & 0.1 & 0.4 & 0.0 & 0.4 & 0.0 & 0.7 & 1.0 & 0.5 & 0.4 & 0.1 & 0.2 & 0.2 & 0.1 & 3.0 & 0.3 & 7.3 \\
\hline \multicolumn{18}{|l|}{ Mining } \\
\hline Drilling oil \& gas wells & 0.0 & 0.0 & 0.0 & 0.0 & 0.0 & 0.0 & 0.0 & 0.0 & 0.0 & 0.0 & 0.0 & 0.0 & 0.0 & 0.0 & 0.0 & 0.0 & 0.0 \\
\hline Oil \& gas extraction & 0.0 & 0.0 & 0.3 & 0.0 & 0.0 & 0.0 & 0.0 & 0.0 & 0.0 & 0.0 & 0.0 & 0.0 & 10.8 & 0.0 & 0.0 & 0.0 & 11.2 \\
\hline Other nonmetallic mineral mining & 0.0 & 0.0 & 0.0 & 0.0 & 0.0 & 0.0 & 0.0 & 0.0 & 0.0 & 0.0 & 0.0 & 0.0 & 0.0 & 0.0 & 0.0 & 0.0 & 0.0 \\
\hline Sand/gravel/clay/refractory mining & 0.0 & 0.0 & 0.4 & 0.0 & 0.9 & 0.0 & 0.0 & 0.0 & 0.0 & 0.0 & 0.0 & 0.0 & 0.1 & 0.0 & 0.0 & 0.0 & 1.5 \\
\hline Stone mining \& quarrying & 0.2 & 0.0 & 0.0 & 0.0 & 0.0 & 0.0 & 0.0 & 0.4 & 0.0 & 0.0 & 0.0 & 0.0 & 0.0 & 0.0 & 0.0 & 0.0 & 0.6 \\
\hline Support activities for oil \& gas & 0.0 & 0.0 & 0.0 & 0.0 & 0.0 & 0.0 & 0.0 & 0.0 & 0.0 & 0.0 & 0.0 & 0.0 & 0.0 & 0.0 & 0.0 & 0.0 & 0.1 \\
\hline Total & 0.3 & 0.0 & 0.8 & 0.0 & 0.9 & 0.0 & 0.0 & 0.4 & 0.0 & 0.0 & 0.0 & 0.0 & 11.0 & 0.0 & 0.0 & 0.0 & 13.4 \\
\hline \multicolumn{18}{|l|}{ Other Crop Farming } \\
\hline All other crop farming & 0.0 & 0.1 & 0.1 & 0.0 & 0.0 & 0.0 & 0.0 & 0.8 & 0.0 & 0.0 & 0.0 & 0.0 & 0.5 & 0.0 & 0.0 & 0.0 & 1.7 \\
\hline Cotton farming & 0.0 & 0.1 & 0.2 & 0.0 & 0.0 & 0.0 & 0.0 & 0.3 & 0.0 & 0.0 & 0.0 & 0.0 & 0.3 & 0.0 & 0.0 & 0.0 & 0.9 \\
\hline Tree nut farming & 0.0 & 0.0 & 0.0 & 0.0 & 0.0 & 0.0 & 0.0 & 0.0 & 0.0 & 0.0 & 0.0 & 0.0 & 0.0 & 0.0 & 0.0 & 0.0 & 0.0 \\
\hline Total & 0.0 & 0.1 & 0.3 & 0.0 & 0.0 & 0.0 & 0.1 & 1.1 & 0.1 & 0.0 & 0.0 & 0.1 & 0.8 & 0.0 & 0.1 & 0.0 & 2.6 \\
\hline
\end{tabular}


Table 13 (continued). Indirect business tax impacts of agriculture and natural resource industries, northwest region of Florida, 2002 data

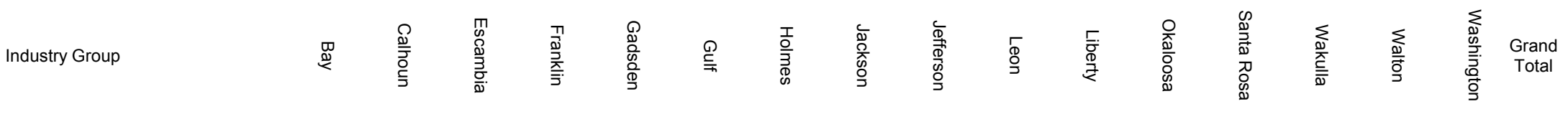

\begin{tabular}{|c|c|c|c|c|c|c|c|c|c|c|c|c|c|c|c|c|c|}
\hline \multirow{2}{*}{$\begin{array}{l}\text { Other Food Product Manufacturing } \\
\text { All other food manufacturing }\end{array}$} & \multirow[b]{2}{*}{0.0} & \multicolumn{16}{|c|}{ (\$Million) } \\
\hline & & 0.0 & 0.0 & 0.0 & 0.0 & 0.0 & 0.0 & 0.0 & 0.0 & 0.0 & 0.0 & 0.0 & 0.0 & 0.0 & 0.0 & 0.0 & 0.0 \\
\hline Bread/bakery, except frozen & 0.0 & 0.0 & 0.0 & 0.0 & 0.0 & 0.0 & 0.0 & 0.0 & 0.0 & 0.0 & 0.0 & 0.0 & 0.1 & 0.0 & 0.0 & 0.0 & 0.2 \\
\hline Coffee \& tea manufacturing & 0.0 & 0.0 & 0.0 & 0.0 & 0.0 & 0.0 & 0.0 & 0.0 & 0.0 & 0.0 & 0.0 & 0.0 & 0.0 & 0.0 & 0.0 & 0.0 & 0.0 \\
\hline Mayonnaise/dressing/sauce mfg & 0.0 & 0.0 & 0.0 & 0.0 & 0.0 & 0.0 & 0.0 & 0.0 & 0.0 & 0.0 & 0.0 & 0.0 & 0.0 & 0.0 & 0.0 & 0.0 & 0.0 \\
\hline Mixes/dough purchased flour & 0.0 & 0.0 & 0.0 & 0.0 & 0.0 & 0.0 & 0.0 & 0.0 & 0.0 & 0.0 & 0.0 & 0.1 & 0.0 & 0.0 & 0.0 & 0.0 & 0.1 \\
\hline Other animal food $\mathrm{mfg}$ & 0.0 & 0.0 & 0.0 & 0.0 & 0.0 & 0.0 & 0.0 & 0.0 & 0.0 & 0.0 & 0.0 & 0.0 & 0.0 & 0.0 & 0.0 & 0.0 & 0.1 \\
\hline Other snack food manufacturing & 0.0 & 0.0 & 0.0 & 0.0 & 0.0 & 0.0 & 0.0 & 0.0 & 0.0 & 0.0 & 0.0 & 0.0 & 0.0 & 0.0 & 0.0 & 0.0 & 0.0 \\
\hline Soft drink \& ice manufacturing & 0.1 & 0.0 & 0.2 & 0.0 & 0.0 & 0.0 & 0.0 & 0.0 & 0.0 & 0.2 & 0.0 & 0.0 & 0.0 & 0.0 & 0.0 & 0.0 & 0.4 \\
\hline Total & 0.1 & 0.0 & 0.2 & 0.0 & 0.0 & 0.0 & 0.0 & 0.0 & 0.0 & 0.2 & 0.0 & 0.1 & 0.1 & 0.0 & 0.0 & 0.0 & 0.8 \\
\hline Non-chocolate Confectionery Mfg & 0.0 & 0.0 & 0.0 & 0.0 & 0.0 & 0.0 & 0.0 & 0.0 & 0.0 & 0.0 & 0.0 & 0.0 & 0.0 & 0.0 & 0.0 & 0.0 & 0.0 \\
\hline Tobacco Farming \& Manufacturing & 0.0 & 0.0 & 0.0 & 0.0 & 0.0 & 0.0 & 0.0 & 0.0 & 0.0 & 0.0 & 0.0 & 0.0 & 0.0 & 0.0 & 0.0 & 0.0 & 0.0 \\
\hline Wildlife (hunting \& trapping) & 0.0 & 0.0 & 0.0 & 0.0 & 0.0 & 0.0 & 0.0 & 0.0 & 0.9 & 0.0 & 0.0 & 0.0 & 0.0 & 0.0 & 0.0 & 0.0 & 0.9 \\
\hline Total Employment Impacts & 10.2 & 1.4 & 18.9 & 1.3 & 8.3 & 0.4 & 1.2 & 5.9 & 2.8 & 3.7 & 1.0 & 2.1 & 14.0 & 0.6 & 3.8 & 1.0 & 76.5 \\
\hline
\end{tabular}

Source: IMPLAN data for Florida counties (2005) 